TRANSACTIONS OF THE

AMERICAN MATHEMATICAL SOCIETY

Volume 361, Number 2, February 2009, Pages 1007-1038

S 0002-9947(08)04679-5

Article electronically published on August 19, 2008

\title{
COMPUTATIONAL TOPOLOGY OF EQUIVARIANT MAPS FROM SPHERES TO COMPLEMENTS OF ARRANGEMENTS
}

\author{
PAVLE V. M. BLAGOJEVIĆ, SINIŠA T. VREĆICA, AND RADE T. ŽIVALJEVIĆ
}

\begin{abstract}
The problem of the existence of an equivariant map is a classical topological problem ubiquitous in topology and its applications. Many problems in discrete geometry and combinatorics have been reduced to such a question and many of them resolved by the use of equivariant obstruction theory. A variety of concrete techniques for evaluating equivariant obstruction classes are introduced, discussed and illustrated by explicit calculations. The emphasis is on $D_{2 n}$-equivariant maps from spheres to complements of arrangements, motivated by the problem of finding a 4 -fan partition of 2 -spherical measures, where $D_{2 n}$ is the dihedral group. One of the technical highlights is the determination of the $D_{2 n}$-module structure of the homology of the complement of the appropriate subspace arrangement, based on the geometric interpretation for the generators of the homology groups of arrangements.
\end{abstract}

\section{INTRODUCTION}

1.1. Computational equivariant topology. One of the objectives of this paper is to identify the problem of calculating the topological obstructions for the existence of equivariant maps as one of the problems paradigmatic for computational topology. Given a group $G$, a $G$-equivariant map $f: X \rightarrow Y$ between two $G$-spaces is a symmetry preserving map, i.e. a map satisfying the condition $f(g \cdot x)=g \cdot f(x)$. In applications in discrete and computational geometry, $X$ is usually a manifold of all "feasible" configurations (the configurations space) while $Y$ is typically a complement of a real, affine subspace arrangement (the test space).

The problem of calculating/evaluating the complexity of equivariant obstructions has many aspects relevant for computational topology, including the following.

$\left(A_{1}\right)$ The (non)existence of an equivariant map is an essential ingredient in the application of the configuration space/test map scheme [36], 19] which has proven to be a very effective tool in solving combinatorial or discrete geometric problems of relevance to computing and analysis of algorithms, 19, 37.

$\left(A_{2}\right)$ The existence of an equivariant map can often be interpreted as a problem of mapping an object (often a cell of some dimension) to a Euclidean space, subject to some boundary constraints and avoiding some obstacles (arrangement of subspaces). This aspect can be seen as a relative of the

Received by the editors June 10, 2005 and, in revised form, April 3, 2006 and May 7, 2007.

2000 Mathematics Subject Classification. Primary 52A37, 55S35; Secondary 55M35.

Key words and phrases. Partition of measures, $k$-fans, equivariant obstruction theory.

This research was supported by grants 144018 and 144026 of the Serbian Ministry of Science, Technology and Ecology.

(C)2008 American Mathematical Society Reverts to public domain 28 years from publication 
motion planning problem from robotics (the case of a 1-dimensional cell). The existence of an equivariant map is equivalent to the problem of sectioning a (vector) bundle, and the condition of avoiding obstacles is translated into the question of sectioning a bundle subject to some additional constraints (relations). The latter problem is of utmost importance in many areas of mathematics, including Gromov convex integration theory [16], combinatorial geometry on vector bundles 35, etc.

Conclusion. Both aspects of the problem of existence of an equivariant map are of theoretical interest. If an equivariant map exists, one should be able to describe and evaluate the complexity of an algorithm that constructs such a map. If an equivariant map does not exist, one is interested in the evaluation and complexity of the associated obstruction. One way to achieve both goals is to develop an "effective" obstruction theory. This is of particular interest in the context of $\left(A_{2}\right)$ where such a theory would allow effective placements of objects into an environment with obstacles, subject to some boundary constraints.

1.2. This paper. We use a well-known problem in discrete and computational geometry (Problem 2.1] in the case $k=4, m=2$ ) as a motivation, and an associated question from equivariant topology (Problem 2.3) as a point of departure to illustrate many aspects, both theoretical and computational, of the general existence of an equivariant map problem. A variety of techniques are introduced and discussed with the emphasis on concrete and explicit calculations. This eventually leads to an almost exhaustive analysis of when such maps do or do not exist in this particular case of interest. The scope and character of these results is illustrated by the following summary of results subsumed by Theorem 4.10.

Summary. Let $G=Q_{4 n}$ be the generalized quaternion group and $M(\alpha)$ the complement of the arrangement $\mathcal{A}(\alpha)$ introduced in Section 2.2

(A) If $\alpha=(p, p, p, p)$ or $\alpha=(p, p, p, 2 p)$, then there are no $Q_{4 n}$-equivariant maps $F: S^{3} \rightarrow M(\alpha)$.

(B) If $\alpha$ is not of the form $(p, q, p+q, p+q)$ or $(p, q, r, p+q), r \notin\{p, p+q\}$, or $(p, p, q, q+r)$, then there exists a $Q_{4 n}$-map $F: S^{3} \rightarrow M(\alpha)$.

(C) If $\alpha=(p, q, p+q, p+q)$ or $\alpha=(p, q, r, p+q), r \notin\{p, p+q\}$, or $\alpha=$ $(p, p, q, q+r)$, the methods of the paper are inconclusive and a further analysis is needed.

The reader is warned however that our analysis of the equivariant problem 2.3 unfortunately does not yield new combinatorial results on the original fan partition problem (problem 2.1). Indeed, all the negative cases covered by part (A) of Theorem 4.10 imply the results already established by the original argument of Bárány and Matoušek [3, 4].

1.3. Obstruction theory in a nutshell. Suppose that $X$ and $Y$ are topological spaces and let $G$ be a group acting on both of them, with the additional assumption that the action on $X$ is free. Furthermore, let $Z$ be a closed, $G$-invariant subspace of $Y$. We focus our attention in this paper on finite groups $G$ and spaces which are simplicial or $C W$-complexes; see [19], [37] or [8] for a glossary of basic topological terms. The problem of deciding if there exists a $G$-equivariant map $f: X \rightarrow Y \backslash Z$ can be approached by two, closely related ways. The first is to build such a map step by step, defining it on the skeletons $X^{(k)}$ one at a time, and attempting to extend 
it to the next skeleton $X^{(k+1)}$. The second is to start with a sufficiently generic, equivariant map $f: X \rightarrow Y$ and, in the case that the singularity $S(f):=f^{-1}(Z)$ is nonempty, try to modify $f$ in an attempt to make the set $S(f)$ vanish. In both approaches there may appear obstructions which prevent us from completing the process, thus showing that such equivariant maps $f: X \rightarrow Y \backslash Z$ do not exist. In the first approach the obstruction is evaluated in the corresponding equivariant cohomology group, while in the second it lies in a "dual" equivariant homology group. When a $G$-action on $X$ is free and the existence of a $G$-map $f$ depends on the primary obstruction, these two methods coincide. The connection is the equivariant Poincaré duality map [8, Theorem 1.4]. In this paper we combine both approaches in the process of identifying the obstruction; see Section 4.1 for some technical details and references.

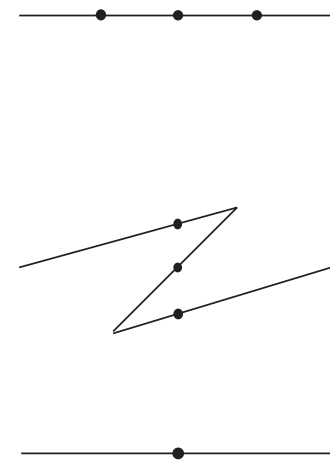

(A)

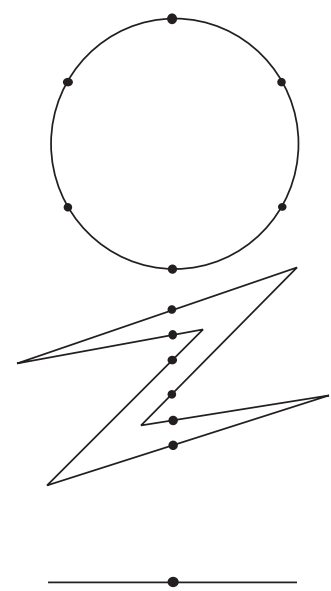

(B)

\section{Figure 1}

As emphasized in the introduction, we focus in this paper on "effective evaluation" of obstruction classes. This is achieved by a careful analysis of:

(a) the singular set $S(f)=f^{-1}(Z)$, which is in this paper just a finite collection $S(f)=\left\{x_{1}, \ldots, x_{m}\right\}$ of points in $X$,

(b) the associated "point classes" $\left\|f\left(x_{i}\right)\right\|$, understood as "weights" of points $x_{i}$, recording the position of $f\left(x_{i}\right)$ in $Z$ and the way a small neighborhood of $x_{i} \in X$ is mapped to a small neighborhood of $f\left(x_{i}\right) \in Y$.

Two naive examples of this procedure are depicted in Figure 1, where $(A)$ illustrates a nonequivariant problem where $X=Y=[-1,+1]$ and $Z=\{0\}$, while (B) corresponds to the case where $G=\mathbb{Z}_{2}, X=S^{1}, Y=[-1,+1]$ and $Z=\{0\}$. However simple, these examples capture some of the main features of the general approach, and the reader should keep in mind that all calculations in this paper follow essentially the same pattern. For example the passage from the figure $(B)$ to the figure $(A)$ illustrates the passage from the $G$-complex $X$ to a "fundamental domain" $A \subset X$, cf. Section 3.1 which allows us to perform the analysis of (a) and (b) in a single, top-dimensional cell $e \subset X$. 


\section{The motivating PRoblem}

2.1. Partition of measures by $k$-fans. A $k$-fan $\mathfrak{p}=\left(x ; l_{1}, l_{2}, \ldots, l_{k}\right)$ on the sphere $S^{2}$ is a point $x$, called the center of the fan, and $k$ great semicircles $l_{1}, \ldots, l_{k}$ emanating from $x$. We always assume counterclockwise enumeration of great semicircles $l_{1}, \ldots, l_{k}$ of a $k$-fan. Sometimes we use the notation $\mathfrak{p}=\left(x ; \sigma_{1}, \sigma_{2}, \ldots, \sigma_{k}\right)$, where $\sigma_{i}$ denotes the open angular sector between $l_{i}$ and $l_{i+1}, i=1, \ldots, k$.

A measure $\mu$ on the sphere $S^{2}$ is proper if $\mu([a, b])=0$ for any circular arc $[a, b] \subset S^{2}$ and $\mu(U)>0$ for each nonempty open set $U \subset S^{2}$.

Let $\mu_{1}, \mu_{2}, \ldots, \mu_{m}$ be proper Borel probability measures on $S^{2}$. All the results can be extended to more general measures, including the counting measures of finite sets; see 3, 34, 35 for related examples.

Let $\left(\alpha_{1}, \alpha_{2}, \ldots, \alpha_{k}\right) \in \mathbb{R}_{>0}^{k}$ be a vector where $\alpha_{1}+\alpha_{2}+\ldots+\alpha_{k}=1$. Following [3] we say that a $k$-fan $\left(x ; l_{1}, \ldots, l_{k}\right)$ is an $\alpha$-partition for the collection $\left\{\mu_{j}\right\}_{j=1}^{m}$ of measures if

$$
(\forall i=1, \ldots, k)(\forall j=1, \ldots, m) \mu_{j}\left(\sigma_{i}\right)=\alpha_{i} .
$$

A vector $\alpha \in \mathbb{R}^{k}$ is called $(m, k)$-admissible if for any collection of $m$ (proper) measures on $S^{2}$, there exists a simultaneous $\alpha$-partition. The collection of all $(m, k)$ admissible vectors is denoted by $\mathcal{A}_{m, k}$. Here is the central problem concerning partitions of measures by $k$-fans.

Problem 2.1 (3], 4, 31]). Describe the set $\mathcal{A}_{m, k}$ or, equivalently, find the integers $m, k$ and vectors $\alpha \in \mathbb{R}^{k}$ such that for any collection $\mathcal{M}=\left\{\mu_{1}, \mu_{2}, \ldots, \mu_{m}\right\}$ of $m$ (proper) measures, there exists an $\alpha$-partition for $\mathcal{M}$.

The analysis given in [3] shows that the most interesting cases of the problem are $(3,2),(2,3)$ and $(2,4)$. It was shown in [3] and [4] that $\left\{\left(\frac{1}{2}, \frac{1}{2}\right),\left(\frac{1}{3}, \frac{2}{3}\right)\right\} \subseteq \mathcal{A}_{3,2}$, $\left\{\left(\frac{1}{3}, \frac{1}{3}, \frac{1}{3}\right),\left(\frac{1}{4}, \frac{1}{4}, \frac{1}{2}\right)\right\} \cup\left\{\left(\frac{a_{1}}{5}, \frac{a_{2}}{5}, \frac{a_{3}}{5}\right)\right\} \subset \mathcal{A}_{2,3}$ and $\left\{\left(\frac{1}{4}, \frac{1}{4}, \frac{1}{4}, \frac{1}{4}\right),\left(\frac{1}{5}, \frac{1}{5}, \frac{1}{5}, \frac{2}{5}\right)\right\} \subseteq \mathcal{A}_{2,4}$.

2.2. The configuration space / test map scheme. The configuration space/ test map scheme is a fairly general method of translating combinatorial geometric problems into topological problems, more precisely problems involving equivariant maps; see [19, 34, 36, 37. It was demonstrated in 3] that the problem of $\alpha$ partitions of spherical measures (Problem 2.1) also admits such a translation. We briefly review this construction focusing on the apparently most challenging case of 4 -fan partitions of two measures on the 2-sphere.

Let $\mu$ and $\nu$ be two Borel, probability measures on $S^{2}$ and $F_{k}$, the space of all $k$-fans on the sphere $S^{2}$. The space $X_{\mu}$ of all $n$-equipartitions of the measure $\mu$ is defined by

$$
X_{\mu}=\left\{\left(x ; l_{1}, \ldots, l_{n}\right) \in F_{n} \mid(\forall i=1, \ldots, n) \mu\left(\sigma_{i}\right)=\frac{1}{n}\right\} .
$$

Every $n$-fan $\left(x ; l_{1}, \ldots, l_{n}\right) \in X_{\mu}$ is completely determined by the pair $\left(x, l_{1}\right)$ or equivalently the pair $(x, y)$, where $y$ is the unit tangent vector to $l_{1}$ at $x$. Thus, the space $X_{\mu}$ is the Stiefel manifold $V_{2}\left(\mathbb{R}^{3}\right)$ of all orthonormal 2-frames in $\mathbb{R}^{3}$. Recall that $V_{2}\left(\mathbb{R}^{3}\right) \cong S O(3) \cong \mathbb{R} P^{3}$.

Let $\mathbb{R}^{n}$ be a Euclidean space with the standard orthonormal basis $e_{1}, e_{2}, \ldots, e_{n}$ and $x_{1}, x_{2}, \ldots, x_{n}$ the associated (dual) coordinate functions. Let $W_{n}$ be the hyperplane $\left\{x \in \mathbb{R}^{n} \mid x_{1}+x_{2}+\ldots+x_{n}=0\right\}$ and suppose that the $\alpha$-vector has the form $\alpha=\left(\frac{a_{1}}{n}, \frac{a_{2}}{n}, \frac{a_{3}}{n}, \frac{a_{4}}{n}\right) \in \frac{1}{n} \mathbb{N}^{4} \subset \mathbb{Q}^{4}$ where $a_{1}+a_{2}+a_{3}+a_{4}=n$. Then the test 
map $F_{\nu}: X_{\mu} \rightarrow W_{n} \subset \mathbb{R}^{n}$ for the measure $\nu$ is defined by

$$
F_{\nu}(x, y)=F_{\nu}\left(x ; l_{1}, \ldots, l_{n}\right)=\left(\nu\left(\sigma_{1}\right)-\frac{1}{n}, \nu\left(\sigma_{2}\right)-\frac{1}{n}, \ldots, \nu\left(\sigma_{n}\right)-\frac{1}{n}\right) .
$$

The natural group of symmetries arising in this problem is the dihedral group $D_{2 n}$. It is interesting and often useful to forget a part of the structure and study the associated problem relative to other subgroups $G \subset D_{2 n}$. The associated test subspace is in that case the space $D_{G}(\alpha)=D(\alpha):=\bigcup \mathcal{A}(\alpha) \subset W_{n}$ defined as the union of the smallest $G$-invariant linear subspace arrangement $\mathcal{A}(\alpha)$ in $\mathbb{R}^{n}$ containing the linear subspace $L(\alpha) \subset W_{n}$, where

$$
L(\alpha):=\left\{x \in \mathbb{R}^{n} \mid z_{1}(x)=z_{2}(x)=z_{3}(x)=z_{4}(x)=0\right\},
$$

and

$$
\begin{array}{ll}
z_{1}(x)=x_{1}+x_{2}+\ldots+x_{a_{1}}, & z_{2}(x)=x_{a_{1}+1}+\ldots+x_{a_{1}+a_{2}}, \\
z_{3}(x)=x_{a_{1}+a_{2}+1}+\ldots+x_{a_{1}+a_{2}+a_{3}}, & z_{4}(x)=x_{a_{1}+a_{2}+a_{3}+1}+\ldots+x_{n} .
\end{array}
$$

Obviously $\operatorname{dim} L(\alpha)=n-4$ and $\operatorname{codim}_{W_{n}} L(\alpha)=3$ since for $x \in W_{n}$, one of the equations $z_{i}(x)=0$ is redundant.

The action of $D_{2 n}=\left\langle\omega, \eta \mid \omega^{n}=\eta^{2}=1, \omega \eta=\eta \omega^{n-1}\right\rangle$ on the configuration space $X_{\mu}$ and the test space $W_{n}$, respectively, is given by

$$
\begin{array}{ll}
\omega\left(x ; l_{1}, \ldots, l_{n}\right)=\left(x ; l_{2}, \ldots, l_{n}, l_{1}\right), & \eta\left(x ; l_{1}, \ldots, l_{n}\right)=\left(-x ; l_{1}, l_{n}, l_{n-1}, \ldots, l_{2}\right), \\
\omega\left(x_{1}, \ldots, x_{n}\right)=\left(x_{2}, \ldots, x_{n}, x_{1}\right), & \eta\left(x_{1}, \ldots, x_{n}\right)=\left(x_{n}, \ldots, x_{2}, x_{1}\right)
\end{array}
$$

where $\left(x ; l_{1}, \ldots, l_{n}\right) \in X_{\mu}$ and $\left(x_{1}, \ldots, x_{n}\right) \in W_{n}$. $D_{2 n}$ acts also on the complement $M(\alpha)=W_{n} \backslash D(\alpha)$ since $\mathcal{A}(\alpha)$ is the smallest $D_{2 n}$-invariant, linear subspace arrangement which contains the linear subspace $L(\alpha)$. Note that the $D_{2 n}$-action on the configuration space $X_{\mu}$ is free. The test map $F_{\nu}$ is a $D_{2 n}$-equivariant. The configuration space $X_{\mu}$ is $D_{2 n}$-homeomorphic to the Stiefel manifold $V_{2}\left(\mathbb{R}^{3}\right)$, which as a $D_{2 n}$-space has the action described by

$$
\omega(x, y)=\left(x, R_{x}\left(\frac{2 \pi}{n}\right)(y)\right), \eta(x, y)=(-x, y),
$$

where $R_{x}(\theta): \mathbb{R}^{3} \rightarrow \mathbb{R}^{3}$ is the rotation around the axes determined by $x$ through the angle $\theta$. The following lemma is a consequence of this analysis.

Lemma 2.2. Let $\alpha=\left(\frac{a_{1}}{n}, \frac{a_{2}}{n}, \frac{a_{3}}{n}, \frac{a_{4}}{n}\right) \in \frac{1}{n} \mathbb{N}^{4} \subset \mathbb{Q}^{4}$ be a vector such that $a_{1}+$ $a_{2}+a_{3}+a_{4}=n$. Let $G$ be a subgroup of the dihedral group $D_{2 n}$. If there does not exist a $G$-map $F: V_{2}\left(\mathbb{R}^{3}\right) \rightarrow M_{G}(\alpha)$, where $M_{G}(\alpha):=W_{n} \backslash D_{G}(\alpha)$, then for any two measures $\mu$ and $\nu$ on $S^{2}$, there always exists a 4-fan which simultaneously $\alpha$-partitions both $\mu$ and $\nu$. In other words the nonexistence of such a map implies $\alpha \in \mathcal{A}_{2,4}$.

In light of Lemma 2.2, it is natural to focus our attention on the following problem as a problem closely related, albeit not equivalent, to the initial $\alpha$-partition problem.

Problem 2.3. For a given subgroup $G$ of the dihedral group $D_{2 n}$, and the associated $G$-spaces $V_{2}\left(\mathbb{R}^{3}\right)$ and $M_{G}(\alpha)$, find an explicit description of the set

$$
\Lambda_{G}=\left\{\alpha \in \frac{1}{n} \mathbb{N}^{4} \mid \text { there is a } G \text {-equivariant map } F: V_{2}\left(\mathbb{R}^{3}\right) \rightarrow M_{G}(\alpha)\right\} .
$$


2.3. The main problem. The well-known "extension of scalars" equivalence from homological algebra, [10, Section III.3], has a very useful analogue in the category of $G$-spaces and $G$-equivariant maps. This equivalence permits us in some cases to change the group and replace the original $G$-space by a new, more tractable topological space.

Let $X$ be a left $G$-space and $H \triangleleft G$ be a normal subgroup. Then $X / H$ is a $G / H$-space with the action given by $g H(H x)=H(g x)$. A $G / H$-space $Z$ can also be seen as a $G$-space via the quotient homomorphism $\pi: G \rightarrow G / H$ where for $g \in G$ and $z \in Z, g \cdot z:=\pi(g) z$.

Lemma 2.4. Suppose that $X$ and $Z$ are $G$-spaces and $H \triangleleft G$ is a normal subgroup of $G$ which acts trivially on $Z$. Then there exists a $G$-map $\alpha: X \rightarrow Z$ if and only if there exists a $G / H$-map $\beta: X / H \rightarrow Z$, where $X / G$ and $Z / H=Z$ are interpreted as $G / H$-spaces.

Proof. $\Leftarrow$ : Observe that the quotient map $p: X \rightarrow X / H$ is a $G$-map and that the $G / H$-space $X / H$ is a $G$-space via the homomorphism $\pi: G \rightarrow G / H$. If $\beta: X / H \rightarrow$ $Z$ is a $G / H$-map, it is also a $G$-map and the composition $\alpha:=p \circ \beta: X \rightarrow Z$ is a $G$-map. $\Rightarrow$ : Let $\alpha: X \rightarrow Z$ be a $G$-map. Then, since $H$ acts trivially on $Z$, there is a factorization $\alpha=\beta \circ p$ for some $\beta: X / H \rightarrow Z$. We check that $\beta$ is a $G / H$-map,

$$
\beta(g H \cdot H x)=\alpha(g x)=g \alpha(x)=g \beta(H x)=(g H) \beta(H x) .
$$

As an application of Lemma 2.4 we replace the dihedral group $D_{2 n}$ by the generalized quaternion group $Q_{4 n}$ and the original configuration space $V_{2}\left(\mathbb{R}^{3}\right)$ by the sphere $S^{3}$.

Let $S^{3}=S(\mathbb{H})=S p(1)$ be the group of all unit quaternions and let $\epsilon=\epsilon_{2 n}=$ $\cos \frac{\pi}{n}+i \sin \frac{\pi}{n} \in S(\mathbb{H})$ be a root of unity. Group $\langle\epsilon\rangle$ is a subgroup of $S(\mathbb{H})$ of order $2 n$. Then, the generalized quaternion group, [11, p. 253], is the subgroup

$$
Q_{4 n}=\left\{1, \epsilon, \ldots, \epsilon^{2 n-1}, j, \epsilon j, \ldots, \epsilon^{2 n-1} j\right\}
$$

of $S^{3}$ of order $4 n$. Let $H=\left\{1, \epsilon^{n}\right\}=\{1,-1\} \subset Q_{4 n}$. The quotient group $Q_{4 n} / H$ is isomorphic to the dihedral group $D_{2 n}$ of order $2 n$.

Lemma 2.5. There exists a $D_{2 n}$-map $G: V_{2}\left(\mathbb{R}^{3}\right) \rightarrow M(\alpha)$ if and only if there exists a $Q_{4 n}$-map $F: S^{3} \rightarrow M(\alpha)$ where the generalized quaternion group $Q_{4 n}$ acts on $S^{3}$ as a subgroup of $S p(1) \cong S^{3}$, and its action on $W_{n}$ is described by $\epsilon\left(x_{1}, \ldots, x_{n}\right)=\left(x_{2}, \ldots, x_{n}, x_{1}\right)$ and $j\left(x_{1}, \ldots, x_{n}\right)=\left(x_{n}, \ldots, x_{2}, x_{1}\right)$.

Proof. Note that $\epsilon^{n}=j^{2}$ acts trivially on $W_{n}$. Moreover, there is an isomorphism $S^{3} / H \cong \mathbb{R} P^{3} \cong S O(3) \cong V_{2}\left(\mathbb{R}^{3}\right)$. Finally, the $Q_{4 n} / H \cong D_{2 n}$ action on $S^{3} / H \cong$ $V_{2}\left(\mathbb{R}^{3}\right)$ coincides with the $D_{2 n}$-action on $V_{2}\left(\mathbb{R}^{3}\right)$ described in the preceding section. Hence, this lemma is a direct consequence of Lemma 2.4.

Remark 2.6. The $Q_{4 n}$-action on $S^{3}$ is free. The $Q_{4 n}$-action on $W_{n}$ is the restriction of the action on $\mathbb{R}^{n}$ described by

$$
\epsilon \cdot e_{i}=e_{(i+1) \bmod n} \text { and } j \cdot e_{i}=e_{n-i+1},
$$

where $e_{1}, \ldots, e_{n}$ is the standard orthonormal basis in $\mathbb{R}^{n}$.

Lemma 2.5 implies the equivalence of Problem 2.3 with the following problem, which appears to be more tractable from the point of view of equivariant obstruction theory. 
Problem 2.7. For a subgroup $G$ of $Q_{4 n}$ such that $H=\{1,-1\} \subset G \subseteq Q_{4 n}$, describe the set

$$
\Gamma_{G}=\left\{\alpha \in \frac{1}{n} \mathbb{N}^{4} \mid \text { there is a } G \text {-equivariant map } F: S^{3} \rightarrow M_{G}(\alpha)\right\} .
$$

\section{TOPOLOGICAL PRELIMINARIES}

3.1. The geometry of the group $Q_{4 n}$. In this section two $Q_{4 n}$-invariant CWstructures on $S^{3}$ are described. This information is used in the obstruction cocycle computations in Section 4.2 .

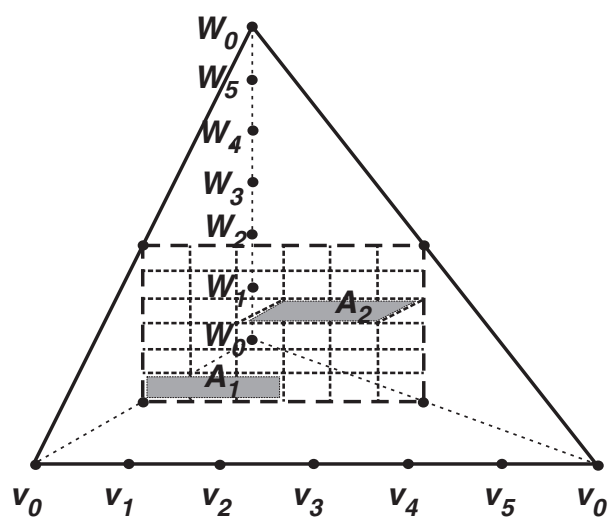

Figure 2. The sphere $S^{3}=P_{2 n}^{(1)} * P_{2 n}^{(2)}$ in the case $n=3$

Let us represent the sphere $S^{3}$ as the join $S^{3}=P_{2 n}^{(1)} * P_{2 n}^{(2)}$ of two $2 n$-gons $P_{2 n}^{(1)}$ and $P_{2 n}^{(2)}$ with respective vertices $v_{i}$ and $w_{j}, 0 \leq i, j \leq 2 n-1$. The case $n=3$ is depicted in Figure 2, where $S^{3}=S^{1} * S^{1}=[0,1] *[0,1] / \approx$ and " $\approx$ " is the equivalence relation on the 3 -simplex $\sigma^{3}:=[0,1] *[0,1]$ arising from the identification $S^{1} \cong[0,1] / 0 \sim 1$. The (big) rectangle represents the torus $T^{2}:=\left\{\frac{1}{2} x+\frac{1}{2} y \mid x, y \in S^{1}\right\}$ subdivided into small rectangles which are the intersections of $T^{2}$ with simplices $\left[v_{i}, v_{i+1} ; w_{j}, w_{j+1}\right]$. Since each point $a=\frac{1}{2} x+\frac{1}{2} y$ of this rectangle uniquely determines the line segment $[x, y] \subset S^{3}$, each subset $A \subset T^{2}$ determines uniquely a set $\widehat{A} \subset S^{3}$ such that $\widehat{A} \cap T^{2}=A$. Let $A_{1}:=\bigcup_{j=0}^{n-1}\left[v_{j}, v_{j+1}\right] \times\left[w_{0}, w_{1}\right]$ be a rectangle in $T^{2}$ and $A_{2}$ a closely related "skew" rectangle; see Figure 2, Then both $\widehat{A}_{1}$ and $\widehat{A}_{2}$ are fundamental domains for the $Q_{4 n}$-action on $S^{3}$, both inducing $Q_{4 n}$-invariant $\mathrm{CW}$-structures on $S^{3}$. Recall that a cell $A$ is a fundamental domain for a free $G$-action of a finite group $G$ on a manifold $M$ if the collection of cells $\{g(A)\}_{g \in G}$ defines a tiling of the manifold in the sense that $M=\bigcup\{g(A)\}_{g \in G}$ and the interiors of cells $g A$ and $h A$ are disjoint for $g \neq h$. Once the fundamental domain $A$ is chosen, its boundary provides all the necessary information about the lower dimensional cells of the associated CW-decomposition.

An analysis of the boundary of the fundamental domain $A_{2}$ shows that the associated CW-structure has only one $Q_{4 n}$-cell $a$ in dimension 0, two $Q_{4 n}$-cells $b$

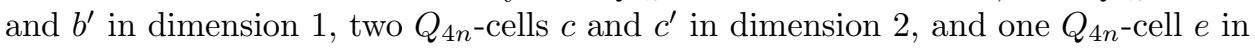
dimension 3 . The reader can easily check that the associated cellular chain complex $\left\{D_{i}\left(S^{3}, \mathbb{Z}\right)\right\}_{i=0}^{3}$ of $Q_{4 n}$-modules coincides with the well-known minimal resolution 
of $\mathbb{Z}$ by free $Q_{4 n}$-modules described on page 253 of the classical monograph [11. More precisely,

$$
0 \rightarrow \mathbb{Z}\left(Q_{4 n}\right) e \stackrel{\partial}{\rightarrow} \mathbb{Z}\left(Q_{4 n}\right) c \oplus \mathbb{Z}\left(Q_{4 n}\right) c^{\prime} \stackrel{\partial}{\rightarrow} \mathbb{Z}\left(Q_{4 n}\right) b \oplus \mathbb{Z}\left(Q_{4 n}\right) b^{\prime} \stackrel{\partial}{\rightarrow} \mathbb{Z}\left(Q_{4 n}\right) a \rightarrow 0,
$$

where

$$
\begin{array}{rlrl}
\partial e=(\epsilon-1) c-(\epsilon j-1) c^{\prime} ; & \partial c=N b-(j+1) b^{\prime} ; & \partial c^{\prime}=(\epsilon j+1) b+(\epsilon-1) b^{\prime} ; \\
\partial b=(\epsilon-1) a ; & \partial b^{\prime}=(j-1) a
\end{array}
$$

and $N=1+\epsilon+\ldots+\epsilon^{n-1} \in \mathbb{Z}\left(Q_{4 n}\right)$. On applying the functor $\operatorname{Hom}_{Q_{4 n}}(\cdot, N)$, where $N:=H_{2}(M ; \mathbb{Z})$ is a $Q_{4 n}$-module, one obtains the sequence (assuming $\mathbb{Z}$ coefficients),

$$
0 \longleftarrow H_{2}(M) \stackrel{\Gamma}{\longleftarrow} H_{2}(M) \oplus H_{2}(M) \longleftarrow H_{2}(M) \oplus H_{2}(M) \longleftarrow H_{2}(M) \longleftarrow 0,
$$

where $\Gamma(p, q)=(\epsilon-1) p-(\epsilon j-1) q$ for $p, q \in H_{2}(M ; \mathbb{Z})$. Now it is not hard to prove

$$
H_{Q_{4 n}}^{3}\left(S^{3}, H_{2}(M ; \mathbb{Z})\right)=H_{2}(M ; \mathbb{Z}) / \operatorname{Im} \Gamma \cong H_{2}(M ; \mathbb{Z})_{Q_{4 n}},
$$

where $H_{2}(M ; \mathbb{Z})_{Q_{4 n}}$ is the group of coinvariants of the $Q_{4 n}$-module $H_{2}(M ; \mathbb{Z})$, [10, Exercise 1, p. 44]. Alternatively, the last result can be seen as a consequence of the equivariant Poincaré duality, [8, Theorem 1.4].

The complex associated with the fundamental domain $A_{1}$, Figure 2, is not as economical as the complex based on the fundamental domain $A_{2}$. Note however that for our purposes this is not a serious disadvantage. Indeed, all we need to know is the intersection of $e_{1}=\operatorname{int}\left(A_{1}\right)$ with the singular set $h^{-1}(\bigcup \mathcal{A}(\alpha))$, for a generic $Q_{4 n}$-equivariant map $h$, and interpret the answer as an element of the group $H_{2}(M ; \mathbb{Z})_{Q_{4 n}}$, cf. Sections 4.2.1 and 4.2.3. On the other hand, the choice of $A_{1}$ has an advantage that its top cell $e_{1}=A_{1}$ is a union of simplices from the original simplicial structure on $S^{3}$, Figure 2 which considerably simplifies the computations performed in Section 4.2 .3 .

3.2. The "point" classes of an arrangement. Assume that orientations on all maximal elements of the arrangement $\mathcal{A}$ and on the ambient space $V$ are given and fixed. The orientations on the orthogonal complements $L_{i}^{\perp}$ are chosen in such a way that together with the orientations on $L_{i}$, they induce the orientation prescribed on $V$.

Let $\mathcal{A}$ be an arrangement of linear subspaces in the $(a+b)$-dimensional Euclidean space $V$ and $\left\{L_{1}, \ldots, L_{k}\right\}$ the set of its elements of maximal dimension. Let us assume that $\operatorname{dim} L_{1}=\cdots=\operatorname{dim} L_{k}=a$. Denote the complement of the arrangement by $M=V \backslash \bigcup \mathcal{A}$.

For a given point $x \in L_{i} \backslash \bigcup_{j \neq i} L_{j}$, let $D_{\varepsilon}(x)=x+D_{\varepsilon}$ be a small disc around $x$, where $D_{\varepsilon}=\left\{y \in L_{i}^{\perp} \mid\langle y, y\rangle \leq \varepsilon\right\}$. "Small" means that $\varepsilon$ is chosen so that $D_{\varepsilon}(x) \cap(\bigcup \mathcal{A})=\{x\}$. We assume that $D_{\varepsilon}(x)$ is oriented, typically by the orientation inherited from already fixed orientation on $L_{i}^{\perp}$. The fundamental class of the pair $\left(D_{\varepsilon}(x), \partial D_{\varepsilon}(x)\right)$ determines a homology class in $H_{b}(V, M ; \mathbb{Z})$, which we denote by $[x]$ and call the point class of $x$. Note that by the Excision axiom, $[x]$ does not depend on $\epsilon$. Moreover, by the Homotopy axiom, $[x]$ does not change if $x$ is moved inside a connected component of $L_{i} \backslash \bigcup_{j \neq i} L_{j}$. Similarly, in light of the isomorphism $H_{b}(V, M ; \mathbb{Z}) \rightarrow H_{b-1}(M ; \mathbb{Z})$, the class $\|x\|:=\partial[x]$, which is also called the point class of $x$, has all these invariance properties as well. 
Let $\hat{V}$ and $\hat{L}_{i}$ stand for spheres obtained by the one-point compactification. By the definition of the point class $\|x\|$ and the associated orientation, the linking number of $\partial D_{\varepsilon}(x)$ and $\hat{L}_{i}$ inside $\hat{V}$ is +1 .

The point classes have the following properties, which can be deduced from Poincaré-Alexander duality and the Goresky-MacPherson formula.

\section{Proposition 3.1.}

(A) For every maximal element $L_{i}$ and every $x \in L_{i} \backslash \bigcup_{j \neq i} L_{j}$ the class $\|x\| \in$ $H_{b-1}(M ; \mathbb{Z})$ is nontrivial.

(B) If the codimension of $L_{i j}:=L_{i} \cap L_{j}$ in $L_{i}$ is greater than 1 for each $j \neq i$ and $x \in L_{i} \backslash \bigcup_{j \neq i} L_{j}$, then the point class $\|x\|$ does not depend on $x \in L_{i}$.

(C) Let $\operatorname{codim}_{L_{i}}\left(L_{i} \cap L_{j}\right)=1$ for some $j \neq i$ where $L_{i}=L_{i}^{1} \cup L_{i}^{2}, L_{j}=L_{j}^{1} \cup L_{j}^{2}$ are decompositions into closed half spaces. If points $x_{1} \in \operatorname{int} L_{i}^{1}$ and $x_{2} \in$ $\operatorname{int} L_{i}^{2}$ then $\left\|x_{1}\right\| \neq\left\|x_{2}\right\|$.

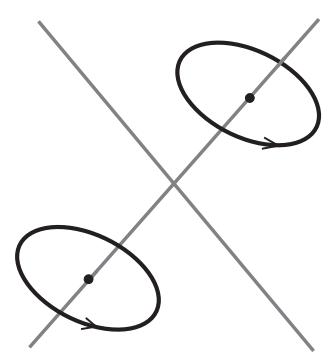

a)

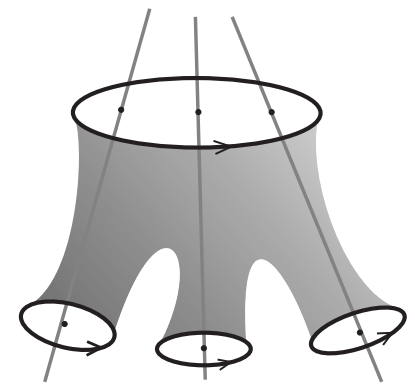

b)

Figure 3 . The point classes

The property of point classes (C) from the previous proposition is illustrated in Figure 3 a.

Proposition 3.2. Assume that $\theta$ is a b-dimensional simplex in $V$ such that $\theta \cap$ $\bigcup \mathcal{A}=\operatorname{int}(\theta) \cap \bigcup \mathcal{A}=\left\{x_{1}, \ldots, x_{k}\right\}$ is a finite set of points and

$(\forall x \in \theta \cap \bigcup \mathcal{A})\left(\exists\right.$ unique $L_{x}$ maximal element of $\left.\mathcal{A}\right)\{x\}=\theta \cap L_{x}$.

The homology class $l \in H_{b-1}(M)$ represented by the boundary $\partial \theta$ of the simplex $\theta$ can be represented as the linear combination of point classes (Figure $3 \mathrm{~b}$ ) in the following way:

$$
l=\mathrm{I}\left(\theta, L_{x_{1}}\right)\left\|x_{1}\right\|+\ldots+\mathrm{I}\left(\theta, L_{x_{k}}\right)\left\|x_{k}\right\|,
$$

where $\mathrm{I}\left(\theta, L_{x_{i}}\right) \in\{-1,+1\}$ is the intersection number of the oriented simplex $\theta$ and $L_{x_{i}}$. Here we assume the already fixed orientation on $L_{x_{i}}$ used in the definition of the point class $\left\|x_{i}\right\|$.

The equality (3.1) is easily deduced from the Excision axiom. It is also implied by the existence of a bordism between $\partial \theta$ and the union of spheres around each $x_{i}$; see Figure 3b. 


\section{Calculation of the obstruction element}

In this section we introduce, following [13, the obstruction exact sequence and apply it to the question of the existence of a $Q_{4 n}$-equivariant map $S^{3} \rightarrow M_{Q_{4 n}}(\alpha)$. The application of the obstruction theory is possible because the $Q_{4 n}$-action on $S^{3}$ is free.

4.1. Obstruction exact sequence. The complement $M=M_{Q_{4 n}}(\alpha)$ of the arrangement $\mathcal{A}(\alpha)$ is 1-connected and consequently 2-simple in the sense that $\pi_{1}(M)$ acts trivially on $\pi_{2}(M)$. Then by [13, Section II.3], and by the Hurewicz theorem, there is an isomorphism $\pi_{2}(M) \cong\left[S^{2}, M\right] \cong H_{2}(M ; \mathbb{Z})$ of $Q_{4 n}$-modules. Thus, the relevant part of the obstruction exact sequence, [13], has the following form:

$$
\left[S^{3}, M\right]_{Q_{4 n}} \stackrel{\theta}{\longrightarrow} \operatorname{Im}\left\{\left[S_{(2)}^{3}, M\right]_{Q_{4 n}} \longrightarrow\left[S_{(1)}^{3}, M\right]_{Q_{4 n}}\right\} \stackrel{\tau}{\longrightarrow} H_{Q_{4 n}}^{3}\left(S^{3}, H_{2}(M ; \mathbb{Z})\right) .
$$

Here $S_{(1)}^{3}$ and $S_{(2)}^{3}$ are respectively the 1 and 2-skeleton of the sphere $S^{3}$, relative to some $Q_{4 n}$-invariant simplicial or CW-structure. Our initial choice is the simplicial structure arising from the join decomposition $S^{3}=P_{2 n}^{(1)} * P_{2 n}^{(2)}$, where both $P_{2 n}^{(1)}$ and $P_{2 n}^{(2)}$ are regular $(2 n)$-sided polygons; see Figure 2. More precisely, the vertices of these polygons are, respectively, $v_{i}:=\epsilon^{i-1} a$ and $w_{i}:=\epsilon^{i} j a, i=0,1, \ldots, 2 n-1$, where $a$ is a fixed complex number, $|a|=1$. The reason we define $v_{i}$ as $\epsilon^{i-1} a$ is technical and will be explained in Section 4.2.1. Since $\left[S_{(1)}^{3}, M\right]_{Q_{4 n}}=\{*\}$ is a oneelement set and $\left[S_{(2)}^{3}, M\right]_{Q_{4 n}}$ is nonempty, the obstruction exact sequence reduces to

$$
\left[S^{3}, M\right]_{Q_{4 n}} \longrightarrow\{*\} \stackrel{\tau}{\longrightarrow} H_{Q_{4 n}}^{3}\left(S^{3}, H_{2}(M ; \mathbb{Z})\right) .
$$

The exactness of this sequence means that the set $\left[S^{3}, M\right]_{Q_{4 n}} \neq \emptyset$ if and only if the element $\tau(*) \in H_{Q_{4 n}}^{3}\left(S^{3}, H_{2}(M ; \mathbb{Z})\right)$ is equal to zero. The class $\tau(*)$ depends only on $M$.

The class $\tau(*)$ can be evaluated by studying the "singular set" of a general position equivariant map, [8, Definition 1.5]. A $Q_{4 n}$-simplicial map $h: S^{3} \rightarrow W_{n}$ has a "general position property" if for each simplex $\sigma$ in $S^{3}$ and any linear space $U$ in the arrangement $\mathcal{A}(\alpha)$ which intersects the $h$-image of $\sigma$, the following conditions are satisfied:

$$
\begin{aligned}
& \text { (A) } \operatorname{dim}(\sigma)=\operatorname{dim}(h(\sigma))=3, \\
& \text { (C) } h(\sigma) \cap U=\{p t\} \subset \operatorname{int}(h(\sigma)) .
\end{aligned}
$$$$
\text { (B) } \operatorname{dim}(U)=n-3 \text {, }
$$

It is not difficult to check that a "generic map" is in general position and that every equivariant, simplicial map can be put in general position by a small perturbation. For each $Q_{4 n}$-map $h: S^{3} \rightarrow W_{n}$ in general position, there is an associated obstruction cocycle

$$
c(h) \in C_{Q_{4 n}}^{3}\left(S^{3}, A\right)=\operatorname{Hom}_{Q_{4 n}}\left(C_{3}\left(S^{3}\right), A\right), \quad[c(h)]=\tau(*),
$$

where $A=H_{2}(M ; \mathbb{Z})$. If $\sigma$ is an oriented 3 -simplex in $S^{3}$, then $c(h)(\sigma) \in H_{2}(M ; \mathbb{Z})$ is the image $h_{*}([\partial(\sigma)])$ of the fundamental class of $\partial(\sigma) \cong S^{2}$ by the map $h_{*}$ : $H_{2}(\partial(\sigma) ; \mathbb{Z}) \rightarrow H_{2}(M ; \mathbb{Z})$. To find an explicit form of the obstruction cocycle $c(h) \in$ $C_{Q_{4 n}}^{3}\left(S^{3}, H_{2}(M ; \mathbb{Z})\right)=\operatorname{Hom}_{Q_{4 n}}\left(C_{3}\left(S^{3}\right), H_{2}(M ; \mathbb{Z})\right)$ we recall that by definition

$$
c(h)(\theta) \neq 0 \Longleftrightarrow h(\theta) \cap(\bigcup \mathcal{A}(\alpha)) \neq \emptyset
$$

and $c(h)(\theta)=h_{*}([\partial \theta]) \in H_{2}(M ; \mathbb{Z})$. 
Before we go further, let us record for future reference an important property of the obstruction cocycle. More details on the restriction and the transfer map can be found in [10, Section III.9].

Lemma 4.1. The cohomology class of the obstruction cocycle $c(h)$ is a torsion element of the group $H_{Q_{4 n}}^{3}\left(S^{3}, H_{2}(M ; \mathbb{Z})\right)$.

Proof. Let $H$ be a subgroup of $Q_{4 n}$. There exists a natural "restriction" map $r: H_{Q_{4 n}}^{3}\left(S^{3}, H_{2}(M ; \mathbb{Z})\right) \rightarrow H_{H}^{3}\left(S^{3}, H_{2}(M ; \mathbb{Z})\right)$, which on the cochain level is just the "forgetful map" sending a $Q_{4 n}$-cochain $c \in C_{Q_{4 n}}^{3}\left(S^{3}, H_{2}(M ; \mathbb{Z})\right)$ to the same cochain interpreted as an $H$-cochain. It follows from the definition of the obstruction cocycle that $r(c(h))$ is the obstruction cocycle for the extension of a general position $H$-map $h$. Moreover there exists a natural map $\tau: H_{H}^{3}\left(S^{3}, H_{2}(M ; \mathbb{Z})\right) \rightarrow$ $H_{Q_{4 n}}^{3}\left(S^{3}, H_{2}(M ; \mathbb{Z})\right)$ in the opposite direction called the transfer map. It is known, [10, Section III.9, Proposition 9.5.(ii)], that the composition of the restriction with the transfer is just multiplication by the index $\left[Q_{4 n}: H\right]$ :

$$
\begin{array}{cccccc}
H_{Q_{4 n}}^{3}\left(S^{3}, H_{2}(M ; \mathbb{Z})\right) & \longrightarrow & H_{H}^{3}\left(S^{3}, H_{2}(M ; \mathbb{Z})\right) & \longrightarrow & H_{Q_{4 n}}^{3}\left(S^{3}, H_{2}(M ; \mathbb{Z})\right) \\
{\left[c(h)_{Q_{4 n}}\right]} & \longmapsto & {\left[c(h)_{H}\right]} & & \longmapsto & {\left[Q_{4 n}: H\right] \cdot\left[c(h)_{Q_{4 n}}\right] .}
\end{array}
$$

Note that if $H$ is the trivial group, the cohomology class of the obstruction cocycle $\left[c(h)_{H}\right]$ is zero. This implies that $[G: H] \cdot\left[c(h)_{Q_{4 n}}\right]=0$ in $H_{Q_{4 n}}^{3}\left(S^{3}, H_{2}(M ; \mathbb{Z})\right)$; i.e., $\left[c(h)_{Q_{4 n}}\right]$ is a torsion element of the group $H_{Q_{4 n}}^{3}\left(S^{3}, H_{2}(M ; \mathbb{Z})\right)$.

Remark 4.2. A consequence of Lemma 4.1 is that special attention should be given to the torsion part of the group $H_{Q_{4 n}}^{3}\left(S^{3}, H_{2}(M ; \mathbb{Z})\right)$.

\subsection{The obstruction cocycle.}

4.2.1. General position $Q_{4 n}$-equivariant maps. Let us start with the description of a general position, simplicial $Q_{4 n}$-map $h: S^{3} \rightarrow W_{n}$, where the sphere has the simplicial structure $S^{3}=P_{2 n}^{(1)} * P_{2 n}^{(2)}$, described in Section 4.1 and depicted in Figure 2. This will be our standard choice of a general position, $Q_{4 n}$-map, fixed throughout the paper. Let $e_{1}, \ldots, e_{n}$ be the standard orthonormal basis in $\mathbb{R}^{n}$ and let $x_{1}, \ldots, x_{n}$ be the associated dual linear functions. Let $\left\{u_{1}, \ldots, u_{n}\right\}$, where $u_{i}=e_{i}-e$ and $e=\frac{1}{n} \sum_{r=1}^{n} e_{r}$, be the vertex set of a regular simplex $\Delta_{n-1}$ in $W_{n}$. If the map $h$ is prescribed in advance on a vertex $t \in P_{2 n}^{(1)}$ and if we require that it is a simplicial $Q_{4 n}$-map, then everything else is completely determined. Let $h(t)=u_{1}$. Then,

$$
\begin{aligned}
h\left(\epsilon^{i} t\right) & =\epsilon^{i} \cdot h(t)=\epsilon^{i} \cdot u_{1}=\epsilon^{i} \cdot\left(e_{1}-e\right)=e_{(i+1) \bmod n}-e=u_{(i+1) \bmod n}, \\
h(j t) & =j \cdot h(t)=j \cdot u_{1}=j \cdot\left(e_{1}-e\right)=e_{n}-e=u_{n}, \\
h\left(\epsilon^{i} j t\right) & =\epsilon^{i} j \cdot h(t)=\epsilon^{i} \cdot\left(e_{n}-e\right)=e_{(i+n) \bmod n}-e=e_{i}-e=u_{i \bmod n} .
\end{aligned}
$$

If the vertices of $P_{2 n}^{(1)}$ and $P_{2 n}^{(2)}$ respectively are $v_{i}:=\epsilon^{i-1} t$ and $w_{i}:=\epsilon^{i} j t$, then the equations (4.2) can be rewritten in a particularly simple form as $h\left(v_{i}\right)=h\left(w_{i}\right)=u_{i}$ for each $i$.

4.2.2. The singular set. To decide whether $h$ is a general position map, it is necessary to determine which simplices $\sigma=\sigma_{1} * \sigma_{2} \subset P_{2 n}^{(1)} * P_{2 n}^{(2)}$ have the property 
that the image $h(\sigma)$ intersects the subspace $L=L(\alpha)$. Note that $h\left(P_{2 n}^{(1)} * P_{2 n}^{(2)}\right) \subseteq$ $\operatorname{sk}_{3}\left(\Delta_{n-1}\right)$, where $\operatorname{sk}_{j}(K)$ is the $j$-skeleton of a simplicial complex $K$. Hence, in order to determine $L \cap h\left(P_{2 n}^{(1)} * P_{2 n}^{(2)}\right)$, we first find the intersection $L \cap \operatorname{sk}_{3}\left(\Delta_{n-1}\right)$. Note that by the defining equations (2.1), for a given increasing sequence of indices $1 \leq i_{1}<i_{2}<i_{3}<i_{4} \leq n$, a simplex $\sigma=\operatorname{conv}\left\{u_{i_{1}}, u_{i_{2}}, u_{i_{3}}, u_{i_{4}}\right\} \in \operatorname{sk}_{3}\left(\Delta_{n-1}\right)$ intersects $L=L(\alpha)$ if and only if

$$
1 \leq i_{1} \leq a_{1}<a_{1}+1 \leq i_{2} \leq a_{2}<a_{2}+1 \leq i_{3} \leq a_{3}<a_{3}+1 \leq i_{4} \leq n .
$$

Moreover, if these conditions are satisfied, then $y=\lambda_{1} u_{i_{1}}+\ldots+\lambda_{4} u_{i_{4}} \in L \cap$ $\operatorname{sk}_{3}\left(\Delta_{n-1}\right)$ if and only if

$$
\lambda_{1}=\frac{a_{1}}{n}, \quad \lambda_{2}=\frac{a_{2}}{n}, \quad \lambda_{3}=\frac{a_{3}}{n}, \quad \lambda_{4}=\frac{a_{4}}{n} .
$$

Suppose that $\sigma$ is in the image $h\left(P_{2 n}^{(1)} * P_{2 n}^{(2)}\right)$. Then, keeping in mind that a typical simplex in $h\left(P_{2 n}^{(1)} * P_{2 n}^{(2)}\right)$ has vertices $v_{k}, v_{k+1}, w_{l}, w_{l+1}$ and that $h\left(v_{i}\right)=h\left(w_{i}\right)=u_{i}$, we conclude that either $\left|i_{2}-i_{1}\right|=1$ and $\left|i_{4}-i_{3}\right|=1$ or, in the opposite case, $\left|i_{3}-i_{2}\right|=1$ and $\left|i_{1}-i_{4}\right|=1 \bmod n$. It can be checked that $L \cap h\left(P_{2 n}^{(1)} * P_{2 n}^{(2)}\right)=$ $\left\{y_{1}, y_{2}\right\}$, where

$$
\begin{aligned}
& y_{1}=\frac{a_{1}}{n} u_{a_{1}}+\frac{a_{2}}{n} u_{a_{1}+1}+\frac{a_{3}}{n} u_{a_{1}+a_{2}+a_{3}}+\frac{a_{4}}{n} u_{a_{1}+a_{2}+a_{3}+1}, \\
& y_{2}=\frac{a_{2}}{n} u_{a_{1}+a_{2}}+\frac{a_{3}}{n} u_{a_{1}+a_{2}+1}+\frac{a_{4}}{n} u_{n}+\frac{a_{1}}{n} u_{1} .
\end{aligned}
$$

This means that there are only two 3 -simplices

$$
\tau_{1}=\left[u_{a_{1}}, u_{a_{1}+1} ; u_{a_{1}+a_{2}+a_{3}}, u_{a_{1}+a_{2}+a_{3}+1}\right] \text { and } \tau_{2}=\left[u_{a_{1}+a_{2}}, u_{a_{1}+a_{2}+1} ; u_{n}, u_{1}\right]
$$

in $h\left(P_{2 n}^{(1)} * P_{2 n}^{(2)}\right)$ which intersect $L$. Note that the point of intersection $y_{i}$ is in the interior of $\tau_{i}$; hence $h$ is a map that always satisfies the first and the third part of the general position condition (4.1) in Section 4.1. The remaining condition that $h(\sigma) \cap U \neq \emptyset$ implies $\operatorname{dim}(U)=n-3$ will be checked separately in Section 4.2.3. cases $(\mathbf{A})$ to $(\mathbf{E})$.

In order to find the singular set $h^{-1}(\bigcup \mathcal{A}(\alpha)) \subseteq S^{3}$, we have to detect all 3simplices $\sigma=\sigma_{1} * \sigma_{2} \subset P_{2 n}^{(1)} * P_{2 n}^{(2)}$ in the sphere $S^{3}$ with the property $\sigma \cap h^{-1}(L) \neq \emptyset$, or in other words the simplices $\sigma=\left[v_{k}, v_{k+1} ; w_{l}, w_{l+1}\right]$ such that either $h(\sigma)=\tau_{1}$ or $h(\sigma)=\tau_{2}$. This leads us to the following four systems of equations:

$$
\begin{array}{lll}
h\left(v_{k}\right)=u_{k \bmod n}=u_{a_{1}} & \text { and } & h\left(w_{l}\right)=u_{l \bmod n}=u_{a_{1}+a_{2}+a_{3}}, \\
h\left(v_{k}\right)=u_{k \bmod n}=u_{a_{1}+a_{2}+a_{3}} & \text { and } & h\left(w_{l}\right)=u_{l \bmod n}=u_{a_{1}}, \\
h\left(v_{k}\right)=u_{k \bmod n}=u_{a_{1}+a_{2}} & \text { and } & h\left(w_{l}\right)=u_{l \bmod n}=u_{n}, \\
h\left(v_{k}\right)=u_{k \bmod n}=u_{n} & \text { and } & h\left(w_{l}\right)=u_{l \bmod n}=u_{a_{1}+a_{2}} .
\end{array}
$$

It turns out that there are 16 , not necessarily different, 3 -simplices $\theta_{1}, \ldots, \theta_{16}$ in $P_{2 n}^{(1)} * P_{2 n}^{(2)}$ which have a nonempty intersection with the singular subset $h^{-1}(L)$, or equivalently which are solutions to one of the four systems of equations (4.4). Here is a complete list where as before $v_{i}$ and $w_{j}$ for $i, j \in\{0, \ldots, 2 n-1\}$ are vertices of 


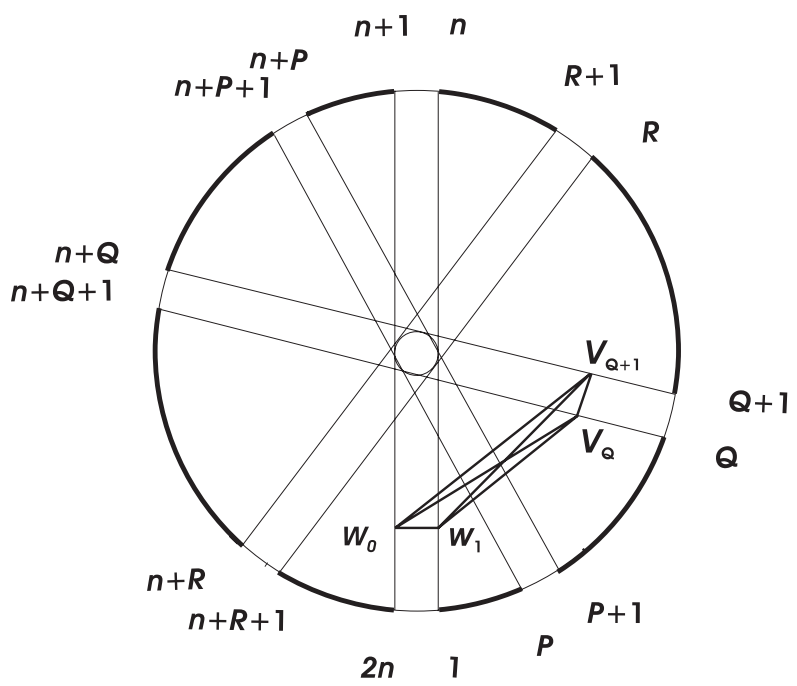

Figure 4 . The simplex $\theta_{11}$ in the sphere $S^{3}=P_{2 n}^{(1)} * P_{2 n}^{(2)}$

$P_{2 n}^{(1)}$ and $P_{2 n}^{(2)}$ respectively and $P=a_{1}, Q=a_{1}+a_{2}, R=a_{1}+a_{2}+a_{3}$. The reader may find Figure 4 useful as a bookkeeping device for generating simplices $\theta_{i}$ :

$$
\begin{array}{ll}
\theta_{1}=\left[v_{P}, v_{P+1} ; w_{R}, w_{R+1}\right], & \theta_{2}=\left[v_{n+P}, v_{n+P+1} ; w_{R}, w_{R+1}\right], \\
\theta_{3}=\left[v_{P}, v_{P+1} ; w_{n+R}, w_{n+R+1}\right], & \theta_{4}=\left[v_{n+P}, v_{n+P+1} ; w_{n+R}, w_{n+R+1}\right], \\
\theta_{5}=\left[v_{R}, v_{R+1} ; w_{P}, w_{P+1}\right], & \theta_{6}=\left[v_{n+R}, v_{n+R+1} ; w_{P}, w_{P+1}\right], \\
\theta_{7}=\left[v_{R}, v_{R+1} ; w_{n+P}, w_{n+P+1}\right], & \theta_{8}=\left[v_{n+R}, v_{n+R+1} ; w_{n+P}, w_{n+P+1}\right], \\
\theta_{9}=\left[v_{Q}, v_{Q+1} ; w_{n}, w_{n+1}\right], & \theta_{10}=\left[v_{n+Q}, v_{n+Q+1} ; w_{n}, w_{n+1}\right], \\
\theta_{11}=\left[v_{Q}, v_{Q+1} ; w_{0}, w_{1}\right], & \theta_{12}=\left[v_{n+Q}, v_{n+Q+1} ; w_{0}, w_{1}\right], \\
\theta_{13}=\left[v_{n}, v_{n+1} ; w_{Q}, w_{Q+1}\right], & \theta_{14}=\left[v_{2 n}, v_{1} ; w_{Q}, w_{Q+1}\right], \\
\theta_{15}=\left[v_{n}, v_{n+1} ; w_{n+Q}, w_{n+Q+1}\right], & \theta_{16}=\left[v_{2 n}, v_{1} ; w_{n+Q}, w_{n+Q+1}\right] .
\end{array}
$$

Then

$$
h\left(\theta_{i}\right)=\tau_{1} \text { if } i \in\{1, \ldots, 8\} \text { and } h\left(\theta_{i}\right)=\tau_{2} \text { if } i \in\{9, \ldots, 16\} .
$$

Simplices $\theta_{1}$ to $\theta_{4}$ arise as the solutions of the first system in (4.4), simplices $\theta_{5}$ to $\theta_{8}$ are solutions to the second system, etc.

4.2.3. The obstruction cocycle $c_{Q_{4 n}}(h)$. By the definition of the obstruction cocycle, for a given 3-simplex $\theta$ of $S^{3}$,

$$
c_{Q_{4 n}}(h)(\theta)=[\partial h(\theta)],
$$

where $[\partial h(\theta)]$ is the homology class determined by the $h$ image of the boundary of $\theta$. Proposition 3.2 implies the formula

$$
c_{Q_{4 n}}(h)(\theta)=\sum_{y \in h(\theta) \cap \cup \mathcal{A}(\alpha)} \mathrm{I}\left(h(\theta), L_{y}\right)\|y\| .
$$

In order to simplify the computation, it is convenient to replace the simplicial complex structure $P_{2 n}^{(1)} * P_{2 n}^{(2)}$ on $S^{3}$ and its chain complex $\left\{C_{i}\left(S^{3}, \mathbb{Z}\right)\right\}_{i=0}^{3}$ by another, more economical $Q_{4 n}$-invariant, cell complex structure on $S^{3}$. In Section 3.1 two such complexes are introduced. The chain complex $\left\{D_{i}\left(S^{3}, \mathbb{Z}\right)\right\}_{i=0}^{3}$, associated 
to the fundamental domain $A_{2}$, is the most economical. However its serious disadvantage is that its top cell $e$ is a "skew cell" in the sense that it is not a union of simplices from the original simplicial decomposition $P_{2 n}^{(1)} * P_{2 n}^{(2)}$. This is the reason why we work with the cell decomposition associated to the fundamental domain $A_{1}$. As noted in Section 3.1 all that appears in calculations is the top-dimensional cell $e_{1}=A_{1}=\bigcup_{j=0}^{n-1}\left[v_{j}, v_{j+1} ; w_{0}, w_{1}\right]$ of this CW-decomposition, so there is no need here to reconstruct the whole $\mathrm{CW}$-complex and the associated chain complex.

The obstruction class computed relative to the cell complex structure based on the cell $e_{1}$, described in Section 3.1, is denoted by $d_{Q_{4 n}}(h)$. To evaluate the obstruction cocycle $d_{Q_{4 n}}(h)$ on the cell $e_{1}$, we are supposed to determine how many simplices of the form $g \cdot \theta_{i}$, where $g \in Q_{4 n}$ and $i \in\{1, \ldots, 16\}$, belong to the cell $e_{1}$. By definition of $e_{1}$, our objective is to determine all elements $g \in Q_{4 n}$ and the corresponding simplices $\theta_{i}$ such that $g \cdot \theta_{i}$ is a simplex of the form $\left[v_{k}, v_{k+1} ; w_{0}, w_{1}\right]$ for some $0 \leq k \leq n-1$. Note that $j v_{k}=w_{1-k}$ and $j w_{l}=v_{n-l+1}$, where the indices in all expressions involving $v_{k}$ and $w_{l}$ are evaluated modulo $2 n$. It follows that

$$
\begin{aligned}
\epsilon^{i}\left[v_{k}, v_{k+1} ; w_{l}, w_{l+1}\right] & =\left[v_{k+i}, v_{k+i+1} ; w_{l+i}, w_{l+i+1}\right], \\
j\left[v_{k}, v_{k+1} ; w_{l}, w_{l+1}\right] & =\left[v_{n-l}, v_{n-l+1} ; w_{-k}, w_{-k+1}\right] .
\end{aligned}
$$

In short, our objective is to inspect the list of all simplices $\theta_{i}, i=1, \ldots, 16$ and determine which group operations turn these simplices into simplices of the form $\left[v_{k}, v_{k+1} ; w_{0}, w_{1}\right]$ for some $0 \leq k \leq n-1$. By direct calculations we arrive at the following list of simplices and the corresponding group elements:

$$
\begin{aligned}
& \sigma_{1}=\left[v_{a_{1}+a_{4}}, v_{a_{1}+a_{4}+1} ; w_{0}, w_{1}\right]=\epsilon^{-a_{1}-a_{2}-a_{3}} \theta_{2}=j \epsilon^{-a_{1}} \theta_{1}, \\
& \sigma_{2}=\left[v_{a_{2}+a_{3}}, v_{a_{2}+a_{3}+1} ; w_{0}, w_{1}\right]=\epsilon^{-a_{1}} \theta_{5}=j \epsilon^{-a_{1}-a_{2}-a_{3}} \theta_{7}, \\
& \sigma_{3}=\left[v_{a_{2}+a_{1}}, v_{a_{2}+a_{1}+1} ; w_{0}, w_{1}\right]=\theta_{11}=j \epsilon^{-a_{1}-a_{2}} \theta_{9}, \\
& \sigma_{4}=\left[v_{a_{3}+a_{4}}, v_{a_{3}+a_{4}+1} ; w_{0}, w_{1}\right]=\epsilon^{-a_{1}-a_{2}} \theta_{13}=j \theta_{14} .
\end{aligned}
$$

Strictly speaking there are two more representations of the form $g \cdot \theta_{j}$ for each of the simplices $\sigma_{i}$. Indeed, if $\sigma_{i}=g \cdot \theta_{j}$, then $\sigma_{i}=g^{\prime} \cdot \theta_{j^{\prime}}$, where $\theta_{j^{\prime}}=\epsilon^{n} \theta_{j}$ and $g^{\prime}=g \epsilon^{n}$.

We conclude that there are four (not necessarily different!) simplices $\sigma_{1}, \sigma_{2}, \sigma_{3}$, $\sigma_{4}$ in the cell $e_{1}$ such that $h\left(\sigma_{i}\right) \cap D(\alpha) \neq \varnothing$ for each $i \in\{1,2,3,4\}$. Our next observation is that for each of the simplices $\sigma_{1}, \sigma_{2}, \sigma_{3}, \sigma_{4}$ the intersection $h\left(\sigma_{i}\right) \cap$ $D(\alpha)$ has two (not necessarily different!) points. More explicitly these points are

$$
\begin{aligned}
& x_{11}=\frac{a_{1}}{n} v_{a_{1}+a_{4}}+\frac{a_{2}}{n} v_{a_{1}+a_{4}+1}+\frac{a_{3}}{n} w_{0}+\frac{a_{4}}{n} w_{1} \in \sigma_{1} \cap h^{-1}\left(\epsilon^{-a_{1}-a_{2}-a_{3}} L\right), \\
& x_{12}=\frac{a_{4}}{n} v_{a_{1}+a_{4}}+\frac{a_{3}}{n} v_{a_{1}+a_{4}+1}+\frac{a_{2}}{n} w_{0}+\frac{a_{1}}{n} w_{1} \in \sigma_{1} \cap h^{-1}\left(j \epsilon^{-a_{1}} L\right), \\
& x_{21}=\frac{a_{3}}{n} v_{a_{2}+a_{3}}+\frac{a_{4}}{n} v_{a_{2}+a_{3}+1}+\frac{a_{1}}{n} w_{0}+\frac{a_{2}}{n} w_{1} \in \sigma_{2} \cap h^{-1}\left(\epsilon^{-a_{1}} L\right), \\
& x_{22}=\frac{a_{2}}{n} v_{a_{2}+a_{3}}+\frac{a_{1}}{n} v_{a_{2}+a_{3}+1}+\frac{a_{4}}{n} w_{0}+\frac{a_{3}}{n} w_{1} \in \sigma_{2} \cap h^{-1}\left(j \epsilon^{-a_{1}-a_{2}-a_{3}} L\right), \\
& x_{31}=\frac{a_{2}}{n} v_{a_{2}+a_{1}}+\frac{a_{3}}{n} v_{a_{2}+a_{1}+1}+\frac{a_{4}}{n} w_{0}+\frac{a_{1}}{n} w_{1} \in \sigma_{3} \cap h^{-1}(L), \\
& x_{32}=\frac{a_{1}}{n} v_{a_{2}+a_{1}}+\frac{a_{4}}{n} v_{a_{2}+a_{1}+1}+\frac{a_{3}}{n} w_{0}+\frac{a_{2}}{n} w_{1} \in \sigma_{3} \cap h^{-1}\left(j \epsilon^{-a_{1}-a_{2}} L\right), \\
& x_{41}=\frac{a_{4}}{n} v_{a_{3}+a_{4}}+\frac{a_{1}}{n} v_{a_{3}+a_{4}+1}+\frac{a_{2}}{n} w_{0}+\frac{a_{3}}{n} w_{1} \in \sigma_{4} \cap h^{-1}\left(\epsilon^{-a_{1}-a_{2}} L\right), \\
& x_{42}=\frac{a_{3}}{n} v_{a_{3}+a_{4}}+\frac{a_{2}}{n} v_{a_{3}+a_{4}+1}+\frac{a_{1}}{n} w_{0}+\frac{a_{4}}{n} w_{1} \in \sigma_{4} \cap h^{-1}(j L) .
\end{aligned}
$$


From here we deduce that

$$
\begin{aligned}
& h\left(\sigma_{1}\right) \cap D(\alpha)=\left(h\left(\sigma_{1}\right) \cap \epsilon^{-a_{1}-a_{2}-a_{3}} L\right) \cup\left(h\left(\sigma_{1}\right) \cap j \epsilon^{-a_{1}} L\right)=\left\{h\left(x_{11}\right), h\left(x_{12}\right)\right\} ; \\
& h\left(\sigma_{2}\right) \cap D(\alpha)=\left(h\left(\sigma_{2}\right) \cap \epsilon^{-a_{1}} L\right) \cup\left(h\left(\sigma_{2}\right) \cap j \epsilon^{-a_{1}-a_{2}-a_{3}} L\right)=\left\{h\left(x_{21}\right), h\left(x_{22}\right)\right\} ; \\
& h\left(\sigma_{3}\right) \cap D(\alpha)=\left(h\left(\sigma_{3}\right) \cap L\right) \cup\left(h\left(\sigma_{3}\right) \cap j \epsilon^{-a_{1}-a_{2}} L\right)=\left\{h\left(x_{31}\right), h\left(x_{32}\right)\right\} ; \\
& h\left(\sigma_{4}\right) \cap D(\alpha)=\left(h\left(\sigma_{4}\right) \cap \epsilon^{-a_{1}-a_{2}} L\right) \cup\left(h\left(\sigma_{4}\right) \cap j L\right)=\left\{h\left(x_{41}\right), h\left(x_{42}\right)\right\}
\end{aligned}
$$

and finally

$$
h\left(e_{1}\right) \cap D(\alpha)=h\left(\left\{x_{11}, x_{12}, x_{21}, x_{22}, x_{31}, x_{32}, x_{41}, x_{42}\right\}\right) .
$$

We have already remarked that some of the points $h\left(x_{i j}\right)$ in (4.5) may coincide; i.e., the cardinality of $h\left(e_{1}\right) \cap D(\alpha)$ can be strictly less than 8 . It turns out that this cardinality is very sensitive to the choice of integers $a_{1}, a_{2}, a_{3}, a_{4}$. Also, this forces us to check if some one of the intersection points in $h(\sigma) \cap D(\alpha)$ is an element of two or more different maximal elements of the arrangement $\mathcal{A}(\alpha)$. If this would be the case, the assumption $h(\sigma) \cap U \neq \emptyset \Rightarrow \operatorname{dim}(U)=n-3$ in (4.1) would be violated. Fortunately the inner symmetries of the arrangement $\mathcal{A}(\alpha)$ will provide that the general position assumption for the map $h$ is satisfied.

(A) Let $a_{2}=a_{4}, a_{1} \neq a_{3}$. Then $\sigma_{1}=\sigma_{3}, \sigma_{2}=\sigma_{4}$ and $x_{11}=x_{32}, x_{12}=x_{31}$, $x_{21}=x_{42}, x_{22}=x_{41}$. These identities imply that

$$
\begin{aligned}
& h\left(x_{11}\right)=h\left(x_{32}\right) \in \epsilon^{-a_{1}-a_{2}-a_{3}} L \cap j \epsilon^{-a_{1}-a_{2}} L, \quad h\left(x_{12}\right)=h\left(x_{31}\right) \in j \epsilon^{-a_{1}} L \cap L, \\
& h\left(x_{22}\right)=h\left(x_{41}\right) \in j \epsilon^{-a_{1}-a_{2}-a_{3}} L \cap \epsilon^{-a_{1}-a_{2}} L, \quad h\left(x_{21}\right)=h\left(x_{42}\right) \in \epsilon^{-a_{1}} L \cap j L .
\end{aligned}
$$

The "symmetry" set equality $\epsilon^{-a_{1}} L=j L$ implies

$$
\begin{aligned}
j \epsilon^{-a_{1}-a_{2}} L & =\epsilon^{(n-1)\left(-a_{1}-a_{2}\right)} j L=\epsilon^{a_{1}+a_{2}} j L=\epsilon^{a_{2}} L=\epsilon^{a_{4}} L=\epsilon^{-a_{1}-a_{2}-a_{3}} L, \\
\epsilon^{-a_{1}} L & =j L, j \epsilon^{-a_{1}} L=\epsilon^{(n-1)\left(-a_{1}\right)} j L=\epsilon^{a_{1}} j L=L, \\
j \epsilon^{-a_{1}-a_{2}-a_{3}} L & =j \epsilon^{a_{4}} L=\epsilon^{(n-1) a_{4}} j L=\epsilon^{-a_{4}-a_{1}} L=\epsilon^{-a_{2}-a_{1}} L .
\end{aligned}
$$

Thus, for each point $h\left(x_{i j}\right)$ there exists a unique, $(n-3)$-dimensional subspace $U \subset D(\alpha)$ such that $h\left(x_{i j}\right) \in D(\alpha)$.

The case when $a_{1}=a_{3}, a_{2} \neq a_{4}$ can be discussed in a similar way with the same conclusion that map $h$ satisfies the general position assumption.

(B) When $a_{2}=a_{4} \neq a_{1}=a_{3}$ the simplices $\sigma_{1}, \sigma_{3}, \sigma_{2}, \sigma_{4}$ coincide and $x_{11}=$ $x_{32}=x_{21}=x_{42}, x_{12}=x_{31}=x_{22}=x_{41}$. In this case,

$$
\begin{aligned}
& h\left(x_{11}\right)=h\left(x_{32}\right)=h\left(x_{21}\right)=h\left(x_{42}\right) \in \epsilon^{-a_{1}-a_{2}-a_{3}} L \cap j \epsilon^{-a_{1}-a_{2}} L \cap \epsilon^{-a_{1}} L \cap j L, \\
& h\left(x_{12}\right)=h\left(x_{31}\right)=h\left(x_{22}\right)=h\left(x_{41}\right) \in j \epsilon^{-a_{1}} L \cap L \cap j \epsilon^{-a_{1}-a_{2}-a_{3}} L \cap \epsilon^{-a_{1}-a_{2}} L .
\end{aligned}
$$

The set of equalities $\epsilon^{a_{1}+a_{2}} L=L$ and $\epsilon^{a_{2}} L=j L$ imply that $h$ is in general position:

$$
\epsilon^{-a_{1}} L=\epsilon^{a_{2}} L=j L=j \epsilon^{a_{1}+a_{2}} L=j \epsilon^{-a_{1}-a_{2}} L=\epsilon^{-a_{1}-a_{2}-a_{3}} L .
$$

(C) Assume $a_{1}=a_{2}=a_{3}=a_{4}$. Then $\sigma_{1}=\sigma_{3}=\sigma_{2}=\sigma_{4}$ and $x_{11}=x_{32}=$ $x_{21}=x_{42}=x_{12}=x_{31}=x_{22}=x_{41}$. Therefore $h\left(x_{11}\right)=\cdots=h\left(x_{41}\right)$ belongs to $\epsilon^{-a_{1}-a_{2}-a_{3}} L \cap j \epsilon^{-a_{1}-a_{2}} L \cap \epsilon^{-a_{1}} L \cap j L \cap j \epsilon^{-a_{1}} L \cap L \cap j \epsilon^{-a_{1}-a_{2}-a_{3}} L \cap \epsilon^{-a_{1}-a_{2}} L$.

Now, with the help of two "symmetry" equalities $\epsilon^{a_{1}} L=L$ and $j L=L$, we obtain $j \epsilon^{-a_{1}-a_{2}-a_{3}} L=j \epsilon^{-a_{1}-a_{2}} L=j \epsilon^{-a_{1}} L=j L=L=\epsilon^{-a_{1}} L=\epsilon^{-a_{1}-a_{2}-a_{3}} L=\epsilon^{-a_{1}-a_{2}} L$.

(D) Let $a_{1}=a_{2} \neq a_{3}=a_{4}$. Then $\sigma_{1}=\sigma_{2}$ and $x_{11}=x_{22}, x_{12}=x_{21}$. In this case,

$h\left(x_{11}\right)=h\left(x_{22}\right) \in \epsilon^{-a_{1}-a_{2}-a_{3}} L \cap j \epsilon^{-a_{1}-a_{2}-a_{3}} L, h\left(x_{12}\right)=h\left(x_{21}\right) \in j \epsilon^{-a_{1}} L \cap \epsilon^{-a_{1}} L$. 
With the equality $j L=\epsilon^{2 a_{3}} L$ we have

$$
\begin{aligned}
j \epsilon^{-a_{1}-a_{2}-a_{3}} L & =j \epsilon^{a_{4}} L=\epsilon^{(n-1) a_{4}} j L=\epsilon^{-a_{4}+2 a_{3}} L=\epsilon^{a_{4}} L=\epsilon^{-a_{1}-a_{2}-a_{3}} L, \\
j \epsilon^{-a_{1}} & =\epsilon^{(n-1)\left(-a_{1}\right)} j L=\epsilon^{a_{1}+2 a_{3}} L=\epsilon^{-a_{1}} L,
\end{aligned}
$$

and conclude that $h$ is in general position.

(E) In all the remaining cases,

$$
h(e) \cap D(\alpha)=\left\{h\left(x_{11}\right), h\left(x_{12}\right), h\left(x_{21}\right), h\left(x_{22}\right), h\left(x_{31}\right), h\left(x_{32}\right), h\left(x_{41}\right), h\left(x_{42}\right)\right\} .
$$

Let $H$ be a $G$-module and $K$ an additive subgroup of $H$ generated by the elements of the form $g \cdot x-x, g \in G, x \in H$. The quotient group $H / K$ is the group of coinvariants [10] of the module $H$ and is denoted by $H_{G}$.

Theorem 4.3. Let $d_{Q_{4 n}}(h) \in C_{Q_{4 n}}^{3}\left(S^{3}, H_{2}(M ; \mathbb{Z})\right)$ denote the obstruction cocycle for the map $h$ defined above.

(A) Let $\alpha=(p, p, p, p)$. Then

$$
d_{Q_{4 n}}(h)(e)=\left\|y_{1}\right\|+K .
$$

(B) Let $\alpha=(p, q, r, q)$ or $\alpha=(q, p, q, r), p \neq r$. Then

$$
\left[d_{Q_{4 n}}(h)(e)\right]=4\left\|y_{1}\right\|+K .
$$

(C) Let $\alpha=(p, q, p, q), p \neq q$. Then

$$
\left[d_{Q_{4 n}}(h)(e)\right]=2\left\|y_{1}\right\|+K .
$$

(D) Let $\alpha=(p, p, q, q)$ or $\alpha=(p, q, q, p), p \neq q$. Then

$$
\left[d_{Q_{4 n}}(h)(e)\right]=2\left\|y_{1}\right\|-2\left\|y_{2}\right\|+K .
$$

(E) In all the remaining cases,

$$
\left[d_{Q_{4 n}}(h)(e)\right]=4\left\|y_{1}\right\|-4\left\|y_{2}\right\|+K .
$$

Proof. Before we calculate obstruction cocycles in all these cases remember that

$$
c_{Q_{4 n}}(h)(\theta)=\sum_{y \in h(\theta) \cap D(\alpha)} \mathrm{I}\left(h(\theta), L_{y}\right)\|y\| .
$$

Also, for every $g \in Q_{4 n}$ there is an identity [14, Proposition 4.5, p. 198]

$$
\mathrm{I}\left(h(g \theta), L_{g \cdot y}\right)=\mathrm{I}\left(h(\theta), L_{y}\right) .
$$

The elementary linear algebra calculation implies that

$$
\mathrm{I}\left(h\left(\theta_{i}\right), L\right)=\left\{\begin{array}{l}
1, i \in\{1, \ldots, 8\} \\
-1, i \in\{9, \ldots, 16\} .
\end{array} \quad h\left(\theta_{i}\right) \cap L=h\left(x_{\theta_{i}}\right)=\left\{\begin{array}{l}
y_{1}, i \in\{1, \ldots, 8\} \\
y_{2}, i \in\{9, \ldots, 16\}
\end{array} .\right.\right.
$$

We assume that the orientation on the subspace $\epsilon^{r} j L$ is induced by the orientation on $L$. If there is a set equality $\epsilon^{r} j L=\epsilon^{k} L$, then we prefer the orientation induced by $\epsilon^{k} L$. From the defining equality for the obstruction cocycle we get

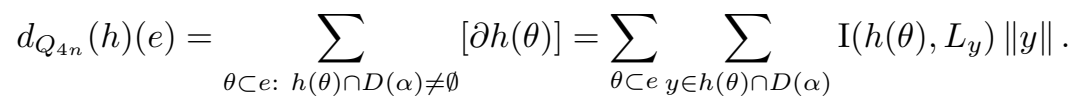


Here $[\partial h(\theta)]$ denotes the homology class determined by the $h$ image of the boundary of the simplex $\theta$. Also, observe that

$$
\begin{aligned}
\left\|h\left(x_{11}\right)\right\| & =\epsilon^{-a_{1}-a_{2}-a_{3}}\left\|y_{1}\right\|, & & \left\|h\left(x_{12}\right)\right\|=j \epsilon^{-a_{1}}\left\|y_{1}\right\|, \\
\left\|h\left(x_{21}\right)\right\| & =\epsilon^{-a_{1}}\left\|y_{1}\right\|, & & \left\|h\left(x_{22}\right)\right\|=j \epsilon^{-a_{1}-a_{2}-a_{3}}\left\|y_{1}\right\|, \\
\left\|h\left(x_{31}\right)\right\| & =\left\|y_{2}\right\|, & & \left\|h\left(x_{32}\right)\right\|=j \epsilon^{-a_{1}-a_{2}}\left\|y_{2}\right\|, \\
\left\|h\left(x_{41}\right)\right\| & =\epsilon^{-a_{1}-a_{2}}\left\|y_{2}\right\|, & & \left\|h\left(x_{42}\right)\right\|=j\left\|y_{2}\right\| .
\end{aligned}
$$

Now we are ready to calculate the obstruction cocycle $d_{Q_{4 n}}(h)(e)$. (A) In the case $a_{1}=a_{2}=a_{3}=a_{4}$, according to previous discussion,

$$
d_{Q_{4 n}}(h)(e)=\left\|h\left(x_{31}\right)\right\|=\left\|y_{1}\right\| .
$$

(B) If $a_{2}=a_{4}$ and $a_{1} \neq a_{3}$, or $a_{1}=a_{3}$ and $a_{2} \neq a_{4}$, then

$$
d_{Q_{4 n}}(h)(e)=\left(\epsilon^{-a_{1}-a_{2}-a_{3}}+j \epsilon^{-a_{1}}+\epsilon^{-a_{1}}+j \epsilon^{-a_{1}-a_{2}-a_{3}}\right)\left\|y_{1}\right\| .
$$

(C) If $a_{2}=a_{4} \neq a_{1}=a_{3}$, then

$$
d_{Q_{4 n}}(h)(e)=\left(\epsilon^{-a_{1}-a_{2}-a_{3}}+j \epsilon^{-a_{1}}\right)\left\|y_{1}\right\| .
$$

(D) If $a_{1}=a_{2} \neq a_{3}=a_{4}$, or $a_{2}=a_{3} \neq a_{1}=a_{4}$, then

$$
d_{Q_{4 n}}(h)(e)=\left(\epsilon^{-a_{1}-a_{2}-a_{3}}+j \epsilon^{-a_{1}}\right)\left\|y_{1}\right\|-\left(1+\epsilon^{-a_{1}-a_{2}}\right)\left\|y_{2}\right\| .
$$

(F) In all the remaining cases, we have

$$
\begin{aligned}
d_{Q_{4 n}}(h)(e)= & \left(\epsilon^{-a_{1}-a_{2}-a_{3}}+j \epsilon^{-a_{1}}+\epsilon^{-a_{1}}+j \epsilon^{-a_{1}-a_{2}-a_{3}}\right)\left\|y_{1}\right\| \\
& -\left(1+j \epsilon^{-a_{1}-a_{2}}+\epsilon^{-a_{1}-a_{2}}+j\right)\left\|y_{2}\right\| .
\end{aligned}
$$

4.3. The cohomology group $H_{Q_{4 n}}^{3}\left(S^{3}, H_{2}(M ; \mathbb{Z})\right)$. The isomorphism

$$
H_{Q_{4 n}}^{3}\left(S^{3}, H_{2}(M ; \mathbb{Z})\right) \cong H_{2}(M ; \mathbb{Z})_{Q_{4 n}}
$$

motivates the study of the $Q_{4 n}$-module structure on $H_{2}(M(\alpha) ; \mathbb{Z})$.

4.3.1. The Homology of $H_{2}(M(\alpha) ; \mathbb{Z})$. The basic idea for computing the homology $H_{2}(M ; \mathbb{Z})$ as an Abelian group is to rely on the Poincaré-Alexander duality and work with the arrangement $\mathcal{A}(\alpha)$ instead of the complement $M$. The complement $M=$ $W_{n} \backslash \bigcup \mathcal{A}(\alpha)$ can be written in the form $S^{n-1} \backslash \bigcup \widehat{\mathcal{A}}(\alpha)$, where $\widehat{\mathcal{A}}(\alpha)$ is the one-point compactification of the arrangement $\mathcal{A}(\alpha)$. There is a sequence of isomorphisms

$$
\begin{aligned}
(4.6) H_{2}(M, \mathbb{Z}) & =H_{2}\left(S^{n-1} \backslash \bigcup \widehat{\mathcal{A}}(\alpha), \mathbb{Z}\right) \quad \text { Poincaré-Alexander duality } \\
& \cong H^{(n-1)-2-1}(\bigcup \widehat{\mathcal{A}}(\alpha), \mathbb{Z}) \quad \text { Universal Coefficient theorem } \\
& \cong \operatorname{Hom}\left(H_{n-4}(\bigcup \widehat{\mathcal{A}}(\alpha), \mathbb{Z}), \mathbb{Z}\right) \oplus \operatorname{Ext}\left(H_{n-5}(\bigcup \widehat{\mathcal{A}}(\alpha), \mathbb{Z}), \mathbb{Z}\right) .
\end{aligned}
$$

The following observation allows us to simplify the computation of the group $\operatorname{Ext}\left(H_{n-5}(\bigcup \widehat{\mathcal{A}}(\alpha), \mathbb{Z}), \mathbb{Z}\right)$ in all cases we are interested in.

Lemma 4.4. If $X$ is a simplicial complex which is homotopy equivalent to a $k$ dimensional complex $Y$, then $H_{k}(X ; \mathbb{Z})$ has no torsion and consequently the group $\operatorname{Ext}\left(H_{k}(X ; \mathbb{Z}) ; \mathbb{Z}\right)$ is a trivial group. 
In the process of computing the homology $H_{2}(M, \mathbb{Z})$ via the isomorphism (4.8), when the Ext-group is trivial, we use the Goresky-MacPherson decomposition (with $\mathbb{Z}$ coefficients)

$$
\begin{aligned}
H_{n-4}(\bigcup \widehat{\mathcal{A}}(\alpha)) & \cong \bigoplus_{V \in P(\alpha)} H_{n-4}\left(\Delta\left(P(\alpha)_{<V}\right) * S^{\operatorname{dim} V}\right) \\
& \cong \bigoplus_{d=0 V \in P(\alpha): \operatorname{dim} V=d}^{n-4} \bigoplus_{n-5-d}\left(\Delta\left(P(\alpha)_{<V}\right)\right),
\end{aligned}
$$

where $P(\alpha)$ is the intersection poset of the arrangement $\mathcal{A}(\alpha)$. Hence, we have to determine

$$
\tilde{H}_{n-5-\operatorname{dim} p}\left(\Delta\left(P(\alpha)_{<V}\right)\right)
$$

for each $V \in P(\alpha)$, where by convention $\tilde{H}_{-1}(\emptyset)=\mathbb{Z}$.

Theorem 4.5. Let $M=W_{n} \backslash \bigcup \mathcal{A}(\alpha)$ be the complement of the minimal $D_{2 n}$ invariant arrangement $\mathcal{A}(\alpha)$ containing the subspace $L(\alpha)$ defined by (2.1) (or equivalently the $Q_{4 n}$-arrangement, where $j^{2}$ acts trivially). Let $\alpha=\left(a_{1}, a_{2}, a_{3}, a_{4}\right) \in$ $\mathbb{N}^{4}$ such that $a_{1}+a_{2}+a_{3}+a_{4}=n$.

(A) If $\alpha=(p, p, p, p)$, then $H_{2}(M ; \mathbb{Z}) \cong \mathbb{Z}^{n / 4}$.

(B) If $\alpha=(p, q, p, q)$ and $p \neq q$, then $H_{2}(M ; \mathbb{Z}) \cong \mathbb{Z}^{n / 2}$.

(C) If $\alpha=(p, p, p, 2 p)$, then $H_{2}(M ; \mathbb{Z}) \cong \mathbb{Z}^{n+\frac{4 n}{5}}$.

(D) If $\alpha=(1,1,1,3)$, then $H_{2}(M ; \mathbb{Z}) \cong \mathbb{Z}^{13}$.

(E) If $\alpha=(q, q, q, p), p \notin\{q, 2 q\}$ and $3 q+p>6$, then $H_{2}(M ; \mathbb{Z}) \cong \mathbb{Z}^{2 n}$.

(F) If $\alpha=(p, q, p, p+q)$ and $p \neq q$, then $H_{2}(M ; \mathbb{Z}) \cong \mathbb{Z}^{2 n}$.

(G) If $\alpha=(p, p, 2 p, 2 p)$, then $H_{2}(M ; \mathbb{Z}) \cong \mathbb{Z}^{2 n+\frac{n}{3}}$.

(H) If $\alpha=(p, q, p+q, p+q)$ and $p \neq q$, then $H_{2}(M ; \mathbb{Z}) \cong$ ?.

(I) If $\alpha=(p, p, q, q)$ and $p \neq q, p \neq 2 q, q \neq 2 p$, then $H_{2}(M ; \mathbb{Z}) \cong \mathbb{Z}^{n}$.

(J) If $\alpha=(p, q, r, q)$ and $r \notin\{q, p, p+q\}$, then $H_{2}(M ; \mathbb{Z}) \cong \mathbb{Z}^{n}$.

(K) If $\alpha=(p, q, r, p+q)$ and $r \notin\{p, p+q\}$, then $H_{2}(M ; \mathbb{Z}) \cong$ ?.

(L) If $\alpha=(p, p, q, q+r)$, and $p \neq q, r \neq 0$, then $H_{2}(M ; \mathbb{Z}) \cong$ ?.

(M) In all the remaining cases $H_{2}(M ; \mathbb{Z}) \cong \mathbb{Z}^{2 n}$.

Remark 4.6. The computation of $H_{2}(M ; \mathbb{Z})$ in the cases $(\mathrm{H}),(\mathrm{K})$ and $(\mathrm{L})$ is probably within reach of the existing methods; however, we leave it as an open problem.

The computation of $H_{2}(M ; \mathbb{Z})$ relies on the isomorphism (4.6) and the fact that the homology group $H_{n-4}(\bigcup \widehat{\mathcal{A}}(\alpha), \mathbb{Z})$ is free. This means that we actually compute $H_{n-4}(\bigcup \widehat{\mathcal{A}}(\alpha), \mathbb{Z})$; i.e., with the help of the Goresky-MacPherson formula we compute $\tilde{H}_{\operatorname{dim} L-\operatorname{dim} p-1}\left(\Delta(P(\alpha))_{<V} ; \mathbb{Z}\right)$ for each $V \in P(\alpha)$.

Proof. (A). In this case there are $\frac{n}{4}$ maximal elements $L, \epsilon L, \ldots, \epsilon^{p-1} L$ of the arrangement $\mathcal{A}(\alpha)$. Since for every $i, j \in\{0, \ldots, p-1\}, i \neq j$,

$$
\operatorname{dim} L-\operatorname{dim}\left(\epsilon^{i} L \cap \epsilon^{j} L\right)>1,
$$

the Goresky-MacPherson formula implies that only maximal elements contribute to $H_{n-4}(\bigcup \widehat{\mathcal{A}}(\alpha), \mathbb{Z})$. The equation (4.7) also guarantees that the assumption of Lemma 4.4 is satisfied, so the corresponding Ext-group is trivial.

(B), (I), (J) and (M). The arrangements for these cases have the same property as in the case (A),

$$
\left(\forall g \in D_{2 n}\right) L \neq g L \Rightarrow \operatorname{dim} L-\operatorname{dim}(L \cap g L)>1 .
$$


Only the number of maximal elements differs. Thus the calculations follow in the footsteps of the case (A).

(C). The arrangement has $n$ maximal elements. The four among them $\epsilon^{p} L$, $\epsilon^{2 p} L, \epsilon^{3 p} L$ and $\epsilon^{4 p} L$ have the property $\operatorname{dim}\left(L \cap \epsilon^{k \cdot p} L\right)=\operatorname{dim} L-1, k=1, \ldots, 4$. In addition,

$$
L \cap \epsilon^{p} L=L \cap \epsilon^{2 p} L=L \cap \epsilon^{3 p} L=L \cap \epsilon^{4 p} L .
$$

Denote the intersection $L \cap \epsilon^{q} L \cap \epsilon^{2 q} L \cap \epsilon^{3 q} L \cap \epsilon^{4 q} L$ by $V$. Then the Hasse diagram of the subposet $\{p \in P(\alpha): \operatorname{dim} p \geq n-5\}$ is depicted in Figure 5, Before using
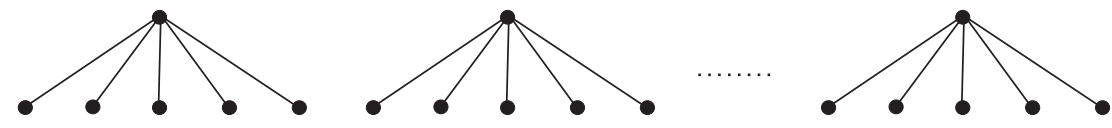

Figure 5. The Hasse diagram for the case (C)

the Goresky-MacPherson decomposition observe that for $n \geq 5$,

$$
(\forall V \in P(\alpha)) \operatorname{dim} V \leq n-6 \Longrightarrow \tilde{H}_{n-5-\operatorname{dim} V}\left(\Delta\left(P(\alpha)_{<V}\right)\right)=0 .
$$

Indeed, for each element $W \in P(\alpha)_{<V}$ such that $\operatorname{dim} W=n-4$ there exists a unique element $V_{W} \in P(\alpha)_{<V}$ with the property $\operatorname{dim} V_{W}=n-5$ and $W<V_{W}$. There is a monotone map $f: P(\alpha)_{<V} \rightarrow P(\alpha)_{<V}-\{W \mid \operatorname{dim} W=n-4\}$ defined by

$$
W \longmapsto\left\{\begin{array}{cl}
W & \text { for } \operatorname{dim} W \leq n-5, \\
V_{W} & \text { for } \operatorname{dim} W=n-4,
\end{array}\right.
$$

which satisfies the conditions of the Quillen fiber lemma. This implies that $f$ induces a homotopy equivalence; hence

$$
\tilde{H}_{n-5-\operatorname{dim} V}\left(\Delta\left(P(\alpha)_{<V}\right)\right)=\tilde{H}_{n-5-\operatorname{dim} V}(\Delta(Q))=0
$$

since $\operatorname{dim} \Delta(Q)<n-5-\operatorname{dim} V$. So, the only relevant part of the poset $P(\alpha)$ for the computation of $H_{n-4}(\bigcup \widehat{\mathcal{A}}(\alpha), \mathbb{Z})$ is the part in Figure 5 . Similarly, this analysis guarantees that the conditions of Lemma 4.4 are satisfied, which again implies that the associated Ext-group is trivial.

(D). The proof follows from the Goresky-MacPherson decomposition and the Hasse diagram of the intersection poset, shown in Figure 6.

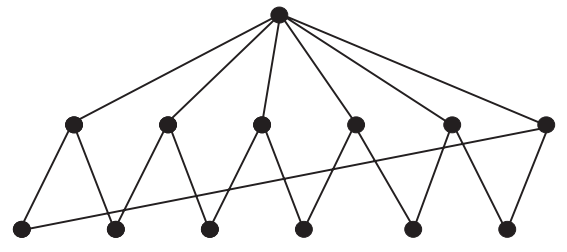

Figure 6. The Hasse diagram for the case (D)

(E). When $\alpha=\left(a_{1}, a_{2}, a_{3}, a_{4}\right)=(q, q, q, p), p \neq 2 q$, the arrangement has $n$ maximal elements and exactly two, $\epsilon^{q} L$ and $\epsilon^{2 q+p} L$, have maximal $L$ intersection, $\operatorname{dim}\left(L \cap \epsilon^{q} L\right)=\operatorname{dim}\left(L \cap \epsilon^{2 q+p} L\right)=\operatorname{dim} L-1$. It is not hard to describe the first 
three levels of the intersection poset $P(\alpha)$. There are $n$ elements $L, \epsilon L, \ldots, \epsilon^{n-1} L$ of dimension $n-4, n$ elements

$$
\begin{aligned}
& L \cap \epsilon^{q} L, \epsilon^{q} L \cap \epsilon^{2 q} L, \ldots, \epsilon^{2 q+p} L \cap L \\
& \epsilon L \cap \epsilon^{q+1} L, \epsilon^{q+1} L \cap \epsilon^{2 q+1} L, \ldots, \epsilon^{2 q+p+1} L \cap \epsilon L ; \\
& \epsilon^{d-1} L \cap \epsilon^{q+d-1} L, \epsilon^{q+d-1} L \cap \epsilon^{2 q+d-1} L, \ldots, \epsilon^{2 q+p+d-1} L \cap \epsilon^{d-1} L
\end{aligned}
$$

of dimension $n-5$ (where $d=(q, n))$ and finally $n$ elements

$$
L \cap \epsilon^{q} L \cap \epsilon^{2 q+p} L, \epsilon\left(L \cap \epsilon^{q} L \cap \epsilon^{2 q+p} L\right), \ldots, \epsilon^{n-1}\left(L \cap \epsilon^{q} L \cap \epsilon^{2 q+p} L\right)
$$

of dimension $n-6$. Thus, the Hasse diagram of the subposet $\{V \in P(\alpha): \operatorname{dim} V \geq$ $n-5\}$ is of the form depicted in Figure 7 . We prove that only this part of
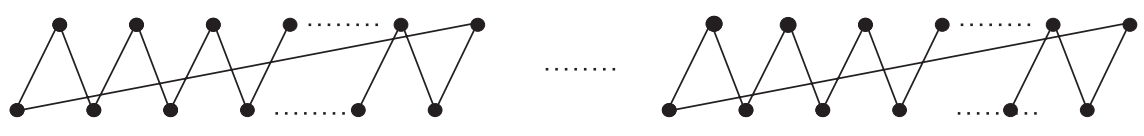

Figure 7. The first Hasse diagram for the case (E)

the Hasse diagram of the intersection poset $P(\alpha)$ contributes to the homology $H_{n-4}(\bigcup \widehat{\mathcal{A}}(\alpha), \mathbb{Z})$. Indeed, for $n>6$,

$$
(\forall V \in P(\alpha)) \operatorname{dim} V \leq n-6 \Longrightarrow \tilde{H}_{n-5-\operatorname{dim} V}\left(\Delta\left(P(\alpha)_{<V}\right)\right)=0 .
$$

Observe that $\operatorname{dim} \Delta\left(P(\alpha)_{<V}\right) \leq n-5-\operatorname{dim} V$. If $\operatorname{dim} \Delta\left(P(\alpha)_{<V}\right)<n-5-\operatorname{dim} V$ we have nothing to do, so we assume that $\operatorname{dim} \Delta\left(P(\alpha)_{<V}\right)=n-5-\operatorname{dim} V$. Let $K=\left\{W \in P(\alpha)_{<V} \mid \operatorname{dim} W \neq n-6\right\}$. Let us show that the inclusion $i: K \hookrightarrow P(\alpha)_{<V}$ satisfies the assumptions of the Quillen fiber lemma. It suffices to check that for every $W=\epsilon^{i}\left(L \cap \epsilon^{q} L \cap \epsilon^{2 q+p} L\right) \in P(\alpha)_{<V}-K$ the order complex $\Delta\left(i^{-1}\left(\left(P(\alpha)_{<V}\right) \leq W\right)\right)$ is contractible. For such a $W=\epsilon^{i}\left(L \cap \epsilon^{q} L \cap \epsilon^{2 q+p} L\right)$, we observe that $\Delta\left(i^{-1}\left(\left(P(\alpha)_{<V}\right)_{\leq W}\right)\right)$ is contractible. Hence, $\Delta\left(P(\alpha)_{<V}\right) \simeq \Delta(K)$. Since $\operatorname{dim} \Delta\left(P(\alpha)_{<V}\right)<n-5-\operatorname{dim} V$, we conclude that $\tilde{H}_{n-5-\operatorname{dim} V}(K)=0$. As before, from the Goresky-MacPherson formula we deduce the following decomposition:

$$
H_{n-4}(\bigcup \widehat{\mathcal{A}}(\alpha) ; \mathbb{Z}) \cong \bigoplus_{d=n-5 V \in P(\alpha): \operatorname{dim} V=d}^{n-4} \bigoplus_{n-5-d}\left(\Delta\left(P(\alpha)_{<V}\right) ; \mathbb{Z}\right) \cong \mathbb{Z}^{n} \oplus \mathbb{Z}^{n}
$$

The case of the group Ext is handled as in previous cases.

(F). There are exactly two maximal elements $\epsilon^{p+q} L$ and $\epsilon^{2 p+q} L$ with the property

$$
\operatorname{dim}\left(L \cap \epsilon^{p+q} L\right)=\operatorname{dim}\left(L \cap \epsilon^{2 p+q} L\right)=\operatorname{dim} L-1 .
$$

Since the subposet $\{V \in P(\alpha): \operatorname{dim} V \geq n-5\}$ of $P(\alpha)$ has the same shape as the appropriate subposet in the proof of part $(E)$, it is not hard to see that the computation for this case goes completely along the lines of the preceding proof.

(G). In this case the arrangement has $n$ maximal elements and two maximal elements, $\epsilon^{2 p} L$ and $\epsilon^{4 p} L$, with the maximal $L$ intersection,

$$
\operatorname{dim}\left(L \cap \epsilon^{2 p} L\right)=\operatorname{dim}\left(L \cap \epsilon^{4 p} L\right)=\operatorname{dim} L-1 .
$$

The elements of $P(\alpha)$ of dimension $n-5$ are: $L \cap \epsilon^{2 p} L, \epsilon\left(L \cap \epsilon^{2 p} L\right), \ldots, \epsilon^{n-1}\left(L \cap \epsilon^{2 p} L\right)$. There are $2 p$ elements of dimension $n-6$ for $n>6$ : $L \cap \epsilon^{2 p} L \cap \epsilon^{4 p} L, \epsilon\left(L \cap \epsilon^{2 p} L \cap\right.$ $\left.\epsilon^{4 p} L\right), \ldots, \epsilon^{2 p}\left(L \cap \epsilon^{2 p} L \cap \epsilon^{4 p} L\right)$ and only one for $n=6$. We use this information to 
draw the Hasse diagram (Figure 8) for the subposet $\{V \in P(\alpha): \operatorname{dim} V \geq n-6\}$, Let us convince ourselves that for $n>6$
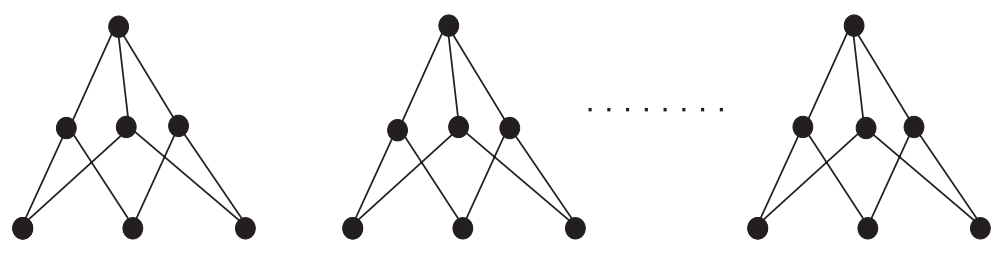

Figure 8. The Hasse diagram for the case $(\mathrm{G})$

$$
(\forall V \in P(\alpha)) \operatorname{dim} V \leq n-7 \Longrightarrow \tilde{H}_{n-5-\operatorname{dim} V}\left(\Delta\left(P(\alpha)_{<V}\right)\right)=0 .
$$

Let $R \stackrel{\text { def }}{=} P(\alpha)_{<V} \cap\{W \in P(\alpha) \mid \operatorname{dim} W=n-6\}=\left\{V_{1}, \ldots, V_{k}\right\}$ where $k \leq 2 p$ and $Q=P(\alpha)_{<V} \cap\{W \in P(\alpha) \mid \operatorname{dim} W \leq n-6\}$. Since for every element $W \in$ $P(\alpha)_{<V} \cap\{W \in P(\alpha) \mid \operatorname{dim} W>n-6\}$ there exists a unique element $V_{W} \in R$ such that $W<V_{W}$, we can define a monotone map $f: P(\alpha)_{<V} \rightarrow Q$ by the formula

$$
f(W)=\left\{\begin{array}{l}
W, \operatorname{dim} W \leq n-6 \\
V_{W}, \operatorname{dim} W>n-6 .
\end{array}\right.
$$

Again the Quillen fiber lemma implies that $f$ induces a homotopy equivalence $\Delta\left(P(\alpha)_{<V}\right) \simeq \Delta(Q)$. Indeed, for each $W \in Q$ the poset $f^{-1}\left(\left(P(\alpha)_{<V}\right)_{\leq W}\right)$ has a maximum; hence $\Delta\left(f^{-1}\left(\left(P(\alpha)_{<V}\right)_{\leq W}\right)\right)$ is contractible. It follows from $\operatorname{dim} \Delta(Q)<n-5-\operatorname{dim} V$ that

$$
\tilde{H}_{n-5-\operatorname{dim} V}\left(\Delta\left(P(\alpha)_{<V}\right)\right)=\tilde{H}_{n-5-\operatorname{dim} V}(\Delta(Q))=0 .
$$

From here we infer that the Goresky-MacPherson formula decomposition reduces to the first three levels of the Hasse diagram of $P(\alpha)$ and

$$
H_{n-4}(\bigcup \widehat{\mathcal{A}}(\alpha), \mathbb{Z}) \cong \mathbb{Z}^{n} \oplus \mathbb{Z}^{n} \oplus \mathbb{Z}^{\frac{n}{3}} .
$$

4.3.2. The $Q_{4 n}$-module structure on $H_{2}(M(\alpha) ; \mathbb{Z})$. To detect the $Q_{4 n}$-module structure on $H_{2}(M(\alpha) ; \mathbb{Z})$ we have to understand how the isomorphisms

$$
\left.H_{2}(M, \mathbb{Z}) \stackrel{\phi}{\rightarrow} \operatorname{Hom}\left(H_{n-4}(\bigcup \widehat{\mathcal{A}}(\alpha), \mathbb{Z}), \mathbb{Z}\right) \stackrel{\psi}{\rightarrow} H_{n-4}(\bigcup \widehat{\mathcal{A}}(\alpha), \mathbb{Z})\right),
$$

valid if $\operatorname{Ext}\left(H_{n-5}(\bigcup \widehat{\mathcal{A}}(\alpha), \mathbb{Z}), \mathbb{Z}\right)=0$, respect the action of the group. In essence the isomorphism $\phi$ is given by the linking number of homology classes in the sphere $\hat{W}_{n}$. The Poincaré-Alexander duality map is a $G$-map up to an "orientation character". The duality map is defined as the map $X \mapsto X \cap O$, where $O$ is a fundamental class of $S^{n-1}$ and " $\cap$ " the usual "cap"-pairing between homology and cohomology. So in order to describe the $G$-action via the Poincaré-Alexander duality (4.6), one has to take into account the associated "orientation character", i.e. the way $O$ transforms by the action of $G$. Since the action of $G$ on $W_{n} \subset S^{n-1}$ is linear, $g(O)=\operatorname{det}(g) \cdot O$, for each $g$ in $G$. The isomorphism of the Universal Coefficient Theorem (4.6) is a 
$G$-map when the action on $\operatorname{Hom}\left(H_{n-4}(\bigcup \widehat{\mathcal{A}}(\alpha), \mathbb{Z}), \mathbb{Z}\right)$ is given by

$$
(g \cdot \xi)(x)=\xi\left(g^{-1} \cdot x\right)
$$

for $g \in Q_{4 n}, \xi \in \operatorname{Hom}\left(H_{n-4}(\bigcup \widehat{\mathcal{A}}(\alpha), \mathbb{Z}), \mathbb{Z}\right)$ and $x \in H_{n-4}(\bigcup \widehat{\mathcal{A}}(\alpha), \mathbb{Z})$. The map $\phi$,

$$
H_{2}(M, \mathbb{Z}) \rightarrow \operatorname{Hom}\left(H_{n-4}(\bigcup \widehat{\mathcal{A}}(\alpha), \mathbb{Z}), \mathbb{Z}\right),
$$

is a $Q_{4 n}$ isomorphism if the "*"-action on $\operatorname{Hom}\left(H_{n-4}(\bigcup \widehat{\mathcal{A}}(\alpha), \mathbb{Z}), \mathbb{Z}\right)$ is given by

$$
(g * \xi)(x):=\operatorname{det}(g) \xi\left(g^{-1} \cdot x\right)=\operatorname{det}\left(g^{-1}\right) \xi\left(g^{-1} \cdot x\right) .
$$

Therefore,

$$
H_{2}(M, \mathbb{Z})_{Q_{4 n}} \cong \operatorname{Hom}\left(H_{n-4}(\bigcup \widehat{\mathcal{A}}(\alpha), \mathbb{Z}), \mathbb{Z}\right)_{Q_{4 n}} .
$$

Remark 4.7. As we have seen in Theorem 4.5 the homology $H_{n-4}(\bigcup \widehat{\mathcal{A}}(\alpha), \mathbb{Z})$ is free. Therefore, there is an isomorphism of abelian groups

$$
\operatorname{Hom}\left(H_{n-4}(\bigcup \widehat{\mathcal{A}}(\alpha), \mathbb{Z}), \mathbb{Z}\right) \cong H_{n-4}(\bigcup \widehat{\mathcal{A}}(\alpha), \mathbb{Z}) .
$$

The isomorphism (4.12) does not induce an isomorphism of $Q_{4 n}$-module structures given by ambient or modified actions. This fact is an extremely important detail [26, 24]. Therefore, we are forced to work inside the $Q_{4 n}$-module $\operatorname{Hom}\left(H_{n-4}(\bigcup \widehat{\mathcal{A}}(\alpha), \mathbb{Z}), \mathbb{Z}\right)$ or directly inside $H_{2}(M, \mathbb{Z})$. Nevertheless, there are situations when these two module structures coincide. For example, let $G$ be a finite group and $H$ a subgroup. Let the $G$-module structure on $\xi \in \operatorname{Hom}(\mathbb{Z}[G / H], \mathbb{Z})$ be defined by $(g \cdot \xi)(x):=\xi\left(g^{-1} \cdot x\right)$, for $x \in \mathbb{Z}[G / H]$. Then there is a $G$-module isomorphism

$$
\operatorname{Hom}(\mathbb{Z}[G / H], \mathbb{Z}) \cong \mathbb{Z}[G / H] .
$$

Theorem 4.8. Let $M=W_{n} \backslash \bigcup \mathcal{A}(\alpha)$ be the complement of the minimal $D_{2 n^{-}}$ invariant arrangement $\mathcal{A}(\alpha)$ containing the subspace $L(\alpha)$ defined by (2.1) (or equivalently the $Q_{4 n}$-arrangement, where $j^{2}$ acts trivially). Let $\alpha=\left(a_{1}, a_{2}, a_{3}, a_{4}\right) \in$ $\mathbb{N}^{4}$ such that $a_{1}+a_{2}+a_{3}+a_{4}=n$.

(A) If $\alpha=(p, p, p, p)$, then $H_{2}(M ; \mathbb{Z}) \cong \mathbb{Z}\left[Q_{4 n}\right] \xi /\left\langle\left(\epsilon^{p}+1\right) \xi,(j-1) \xi\right\rangle$.

(B) If $\alpha=(p, q, p, q)$ and $p \neq q$, then

$$
H_{2}(M ; \mathbb{Z}) \cong \mathbb{Z}\left[Q_{4 n}\right] \xi /\left\langle\left(\epsilon^{p+q}-1\right) \xi,\left(j^{2}-1\right) \xi,\left(\epsilon^{-q} j+1\right) \xi\right\rangle .
$$

(C) If $\alpha=(p, p, p, 2 p)$ and

$\left.K=\left\langle 1+\epsilon^{p}+\epsilon^{2 p}+\epsilon^{3 p}+\epsilon^{4 p}+j+\epsilon^{p} j+\epsilon^{2 p} j+\epsilon^{3 p} j+\epsilon^{4 p} j\right) \xi,\left(j^{2}-1\right) \xi,\left(\epsilon^{n}-1\right) \xi\right\rangle$, then

$$
H_{2}(M ; \mathbb{Z}) \cong \mathbb{Z}\left[Q_{4 n}\right] \xi / K
$$

(D) If $\alpha=(1,1,1,3)$ and

$$
K=\left\langle\left(\epsilon^{-3} j+1\right) \xi,\left(\epsilon^{6}-1\right) \xi,\left(1+\epsilon^{-2} j\right) \eta,\left(\epsilon^{6}-1\right) \eta,(1-j) \zeta,(1+\epsilon) \zeta\right\rangle,
$$

then

$$
H_{2}(M ; \mathbb{Z}) \cong \mathbb{Z}\left[Q_{4 n}\right] \xi \oplus \mathbb{Z}\left[Q_{4 n}\right] \eta \oplus \mathbb{Z}\left[Q_{4 n}\right] \zeta / K .
$$

(E) If $\alpha=(q, q, q, p), p \notin\{q, 2 q\}$ and $3 q+p>6$, then

$H_{2}(M ; \mathbb{Z}) \cong \mathbb{Z}\left[Q_{4 n}\right] \xi \oplus \mathbb{Z}\left[Q_{4 n}\right] \eta /\left\langle\left(1+\epsilon^{-p} j\right) \xi,\left(\epsilon^{n}-1\right) \xi,\left(j^{2}-1\right) \eta,\left(1+\epsilon^{-2 p-2 q} j\right) \eta\right\rangle$.

(F) If $\alpha=(p, q, p, p+q)$ and $p \neq q$, then

$H_{2}(M ; \mathbb{Z}) \cong \mathbb{Z}\left[Q_{4 n}\right] \xi \oplus \mathbb{Z}\left[Q_{4 n}\right] \eta /\left\langle\left(1+\epsilon^{-p-q} j\right) \xi,\left(\epsilon^{n}-1\right) \xi,\left(j^{2}-1\right) \eta,(1+j) \eta\right\rangle$. 
(G) If $\alpha=(p, p, 2 p, 2 p)$, then $H_{2}(M ; \mathbb{Z}) \cong$ ?

(H) If $\alpha=(p, q, p+q, p+q)$ and $p \neq q$, then $H_{2}(M ; \mathbb{Z}) \cong$ ?.

(I) If $\alpha=(p, p, q, q)$ and $p \neq q, p \neq 2 q, q \neq 2 p$, then

$$
H_{2}(M ; \mathbb{Z}) \cong \mathbb{Z}\left[Q_{4 n}\right] \xi /\left\langle\left(e^{n}-1\right) \xi,\left(\epsilon^{-2 q} j-1\right) \xi\right\rangle .
$$

(J) If $\alpha=(p, q, r, q)$ and $r \notin\{q, p, p+q\}$, then

$$
H_{2}(M ; \mathbb{Z}) \cong \mathbb{Z}\left[Q_{4 n}\right] \xi /\left\langle\left(e^{n}-1\right) \xi,\left(\epsilon^{p} j+1\right) \xi\right\rangle .
$$

(K) If $\alpha=(p, q, r, p+q)$ and $r \notin\{p, p+q\}$, then $H_{2}(M ; \mathbb{Z}) \cong$ ?.

(L) If $\alpha=(p, p, q, q+r)$, and $p \neq q, r \neq 0$, then $H_{2}(M ; \mathbb{Z}) \cong$ ?.

(M) In all the remaining cases, $H_{2}(M ; \mathbb{Z}) \cong \mathbb{Z}\left[Q_{4 n}\right] \xi /\left\langle\left(e^{n}-1\right) \xi\right\rangle$.

Proof. The proof of every instance of the theorem contains three steps:

- study of the $Q_{4 n}$-module structure on $H_{n-4}(\bigcup \widehat{\mathcal{A}}(\alpha), \mathbb{Z})$ with the ambient action,

- study of the $Q_{4 n}$-module structure on $\operatorname{Hom}\left(H_{n-4}(\bigcup \widehat{\mathcal{A}}(\alpha), \mathbb{Z}), \mathbb{Z}\right)$ given by (4.10) using a concrete basis of $H_{n-4}(\bigcup \widehat{\mathcal{A}}(\alpha), \mathbb{Z})$,

- description of the $Q_{4 n}$-module structure of $\operatorname{Hom}\left(H_{n-4}(\bigcup \widehat{\mathcal{A}}(\alpha), \mathbb{Z}), \mathbb{Z}\right)$.

The fact that $j^{2}$ and $\epsilon^{n}$ are acting trivially on $W_{n}$ is affecting all module structures we study.

(A) Let $x \in H_{n-4}(\bigcup \widehat{\mathcal{A}}(\alpha), \mathbb{Z})$ be the homology class induced by the subspace $L$. The set equality $L=\epsilon^{p} L$ translates to the equality $x=(-1)^{p-1} \epsilon^{p} \cdot x$ in homology, because $\epsilon^{p}$ acts on the orientation of the geometric representative of $x$ by $(-1)^{p-1}$. Indeed, the decomposition $L \oplus L^{\perp}=\mathbb{R}^{n}$ implies the identity

$$
\operatorname{det}\left(\epsilon^{p}\right)=\operatorname{det}\left(\left.\epsilon^{p}\right|_{L}\right) \operatorname{det}\left(\left.\epsilon^{p}\right|_{L^{\perp}}\right) .
$$

To detect the value $\operatorname{det}\left(\left.\epsilon^{p}\right|_{L^{\perp}}\right)$ let us fix

$$
\mathfrak{e}=\left[e_{1}+\cdots+e_{p}, e_{p+1}+\cdots+e_{2 p}, e_{2 p+1}+\cdots+e_{3 p}, e_{3 p+1}+\cdots+e_{n}\right]
$$

as the basis of $L^{\perp}$. Then $\epsilon^{p}$ acts on $\mathfrak{e}$ by permuting elements of the basis in the following way:

$$
\epsilon^{p} \mathfrak{e}=\left[e_{p+1}+\cdots+e_{2 p}, e_{2 p+1}+\cdots+e_{3 p}, e_{3 p+1}+\cdots+e_{n}, e_{1}+\cdots+e_{p}\right] .
$$

Since the permutation of the basis elements is odd, $\operatorname{det}\left(\left.\epsilon^{p}\right|_{L^{\perp}}\right)=-1$. Thus, $\operatorname{det}\left(\epsilon^{p}\right)=(-1)^{p}$ implies that $\operatorname{det}\left(\left.\epsilon^{p}\right|_{L}\right)=(-1)^{p-1}$.

The set equality $L=j L$ in a similar fashion induces the relation $x=(-1)^{\left(\begin{array}{c}n \\ 2\end{array}\right)} j \cdot x$ in $H_{n-4}(\bigcup \widehat{\mathcal{A}}(\alpha), \mathbb{Z})$. Indeed, $j$ acts on the basis $\mathfrak{e}$ of $L^{\perp}$ as the even permutation $\left(\begin{array}{l}1234 \\ 4321\end{array}\right)$. We also inherit relations $\epsilon^{n} \cdot x=x$ and $j^{2} \cdot x=x$.

The set of elements $\Lambda=\left\{x, \epsilon \cdot x, \ldots, \epsilon^{p-1} \cdot x\right\}$ is a basis of the free abelian group $H_{n-4}(\bigcup \widehat{\mathcal{A}}(\alpha), \mathbb{Z})$. Let $\xi \in \operatorname{Hom}\left(H_{n-4}(\bigcup \widehat{\mathcal{A}}(\alpha), \mathbb{Z}), \mathbb{Z}\right)$ be an element given on the basis $\Lambda$ by $\xi(x)=1, \xi\left(\epsilon^{i} \cdot x\right)=0$, for $i \in\{1, \ldots, p-1\}$. Then the set $\left\{\xi, \epsilon * \xi, \ldots, \epsilon^{p-1} * \xi\right\}$ is a basis of the free abelian group $\operatorname{Hom}\left(H_{n-4}(\cup \widehat{\mathcal{A}}(\alpha), \mathbb{Z}), \mathbb{Z}\right)$. From the definition of the "*"-action (4.10), it follows that for $i \in\{0,1, \ldots, p-1\}$,

$$
\begin{aligned}
\epsilon^{p} * \xi\left(\epsilon^{i} \cdot x\right) & =\operatorname{det}\left(\epsilon^{p}\right) \xi\left(\epsilon^{i-p} \cdot x\right)=(-1)^{p} \xi\left(\epsilon^{i+3 p} \cdot x\right)=(-1)^{p} \xi\left(\epsilon^{i} \cdot\left(\epsilon^{3 p} \cdot x\right)\right) \\
& =(-1)^{p} \xi\left(\epsilon^{i} \cdot\left((-1)^{3 p-3} x\right)\right)=(-1)^{4 p-3} \xi\left(\epsilon^{i} \cdot x\right)=-\xi\left(\epsilon^{i} \cdot x\right) .
\end{aligned}
$$


Thus $\epsilon^{p} * \xi=-\xi$. In the same way, for $i \in\{0,1, \ldots, p-1\}$,

$$
\begin{aligned}
j * \xi\left(\epsilon^{i} \cdot x\right) & =\operatorname{det}(j) \xi\left(j^{-1} \epsilon^{i} \cdot x\right)=(-1)^{\left(\begin{array}{c}
n \\
2
\end{array}\right)} \xi\left(j^{3} \epsilon^{i} \cdot x\right)=(-1)^{\left(\begin{array}{c}
n \\
2
\end{array}\right)} \xi\left(\epsilon^{n} j \epsilon^{i} \cdot x\right) \\
& =(-1)^{\left(\begin{array}{c}
n \\
2
\end{array}\right)} \xi\left(\epsilon^{n} \epsilon^{-i} j \cdot x\right)=\xi\left(\epsilon^{4 p-i} \cdot x\right)=\xi\left(\epsilon^{p-i} \cdot\left(\epsilon^{3 p} \cdot x\right)\right) \\
& =-\xi\left(\epsilon^{p-i} \cdot x\right)=\xi\left(\epsilon^{i} \cdot x\right)
\end{aligned}
$$

and consequently $j * \xi=\xi$.

(B) Again $x \in H_{n-4}(\bigcup \widehat{\mathcal{A}}(\alpha), \mathbb{Z})$ denotes the homology class induced by $L$. There are two set equalities, $L=\epsilon^{p+q} L$ and $L=\epsilon^{-q} j L$, besides two usual $L=\epsilon^{n} L$, $L=j^{2} L$. Elements $\epsilon^{p+q}$ and $\epsilon^{-q} j$ act on $L^{\perp}$ as permutations $\left(\begin{array}{l}1234 \\ 3412\end{array}\right)$ and $\left(\begin{array}{l}1234 \\ 3214\end{array}\right)$, respectively. Therefore, in homology,

$$
\epsilon^{p+q} \cdot x=\operatorname{det}\left(\epsilon^{p+q}\right) x, \epsilon^{-q} j \cdot x=-\operatorname{det}\left(\epsilon^{-q} j\right) x, \epsilon^{n} \cdot x=x, j^{2} \cdot x=x .
$$

The set of elements $\Lambda=\left\{x, \epsilon \cdot x, \ldots, \epsilon^{p+q-1} \cdot x\right\}$ is a basis of the free abelian group $H_{n-4}(\bigcup \widehat{\mathcal{A}}(\alpha), \mathbb{Z})$. Let $\xi \in \operatorname{Hom}\left(H_{n-4}(\bigcup \widehat{\mathcal{A}}(\alpha), \mathbb{Z}), \mathbb{Z}\right)$ be an element given on the basis $\Lambda$ by $\xi(x)=1, \xi\left(\epsilon^{i} \cdot x\right)=0$, for $i \in\{1, \ldots, p+q-1\}$. Then the set $\left\{\xi, \epsilon * \xi, \ldots, \epsilon^{p+q-1} * \xi\right\}$ is a basis of the free abelian group $\operatorname{Hom}\left(H_{n-4}(\bigcup \widehat{\mathcal{A}}(\alpha), \mathbb{Z}), \mathbb{Z}\right)$. From the definition of the "*"-action (4.10), it follows that for $i \in\{0,1, \ldots, p+q-1\}$,

$$
\begin{aligned}
\epsilon^{p+q} * \xi\left(\epsilon^{i} \cdot x\right) & =\operatorname{det}\left(\epsilon^{p+q}\right) \xi\left(\epsilon^{i-p-q} \cdot x\right)=\operatorname{det}\left(\epsilon^{p+q}\right) \xi\left(\epsilon^{i+p+q} \cdot x\right) \\
& =\operatorname{det}\left(\epsilon^{p+q}\right) \xi\left(\epsilon^{i} \cdot\left(\epsilon^{p+q} \cdot x\right)\right)=\xi\left(\epsilon^{i} \cdot x\right)
\end{aligned}
$$

and

$$
\begin{aligned}
\epsilon^{-q} j * \xi\left(\epsilon^{i} \cdot x\right) & =\operatorname{det}\left(\epsilon^{-q} j\right) \xi\left(j^{3} \epsilon^{i+q} \cdot x\right)=\operatorname{det}\left(\epsilon^{-q} j\right) \xi\left(\epsilon^{n} j \epsilon^{i+q} \cdot x\right) \\
& =\operatorname{det}\left(\epsilon^{-q} j\right) \xi\left(\epsilon^{n-i-q} j \cdot x\right)=-\xi\left(\epsilon^{n-i} \cdot x\right) \\
& =-\xi\left(\epsilon^{p+q-i} \cdot\left(\epsilon^{p+q} \cdot x\right)\right)=-\xi\left(\epsilon^{p+q-i} \cdot x\right)=-\xi\left(\epsilon^{i} \cdot x\right) .
\end{aligned}
$$

Thus, $\epsilon^{p+q} * \xi=\xi, \epsilon^{-q} j * \xi=-\xi$ and $j^{2} * \xi=\xi$.

(C) Let $I$ be the intersection $L \cap \epsilon^{p} L=L \cap \epsilon^{p} L \cap \epsilon^{2 p} L \cap \epsilon^{3 p} L \cap \epsilon^{4} L$ and $L^{+}$be a closed subspace defined by

$$
L^{+}=\left\{x \in L \mid x_{3 p+1}+\ldots+x_{4 p} \geq 0\right\} .
$$

We make this choice because $y_{1} \in \operatorname{int} L^{+}$( $y_{1}$ is given by 4.3). The "opposite" closed half-space will be denoted by $L^{-}$.

Let $x, y \in H_{n-4}(\bigcup \widehat{\mathcal{A}}(\alpha), \mathbb{Z})$ denote the homology classes induced by $L$ and by $L^{+} \cup \epsilon^{p} L^{+}$. Since $\epsilon^{-2 p} j$ acts on the basis of $L^{\perp}$ as the odd permutation $\left(\begin{array}{l}1234 \\ 3214\end{array}\right)$, the set equality $L=\epsilon^{-2 p} j L$ implies the relation

$$
\left(\epsilon^{-2 p} j\right) \cdot x=-\operatorname{det}\left(\epsilon^{-2 p} j\right) x .
$$

With $j^{2} \cdot x=\epsilon^{n} \cdot x=x$ this competes the set of relations for element $x$.

To describe the action of $Q_{4 n}$ on $y$ let us first choose an orientation on $L^{+} \cup \epsilon^{p} L^{+}$. Let $\mathfrak{e}$ be a basis,

$$
\begin{aligned}
\mathfrak{e}=\left[e_{1}+\cdots+e_{p}, e_{p+1}+\cdots+e_{2 p}, e_{2 p+1}\right. & +\cdots+e_{3 p}, \\
& \left.e_{3 p+1}+\cdots+e_{4 p}, e_{4 p+1}+\cdots+e_{n}\right],
\end{aligned}
$$

of $I^{\perp}$ and $\mathfrak{f}$ a basis of $I$ such that $[\mathfrak{f}, \mathfrak{e}]$ coincides with the prescribed orientation on $W_{n}$. Let $[\mathfrak{e}, v]$ be a basis of $L$ such that vector $v$ points inside $L^{+}$. We assume that $[\mathfrak{e}, v]$ is the prescribed orientation on $L$. Then the geometric class $L^{+} \cup \epsilon^{p} L^{+}$is oriented in the following way: $\left(L^{+},[\mathfrak{f}, v] ; \epsilon^{p} L^{+},\left[-\mathfrak{f}, \epsilon^{p} v\right]\right)$. The $-\mathfrak{f}$ appears because 
the orientations on the boundary of the half-spaces have to be opposite. The element $\epsilon^{p}$ acts on the basis $\mathfrak{e}$ of $I^{\perp}$ by the even permutation $\left(\begin{array}{l}12345 \\ 23451\end{array}\right)$. This implies that $\epsilon^{p}$ acts on the orientation of $I$ by the multiplication with $\operatorname{det}\left(\epsilon^{p}\right)$. The geometric representatives of classes $y, \epsilon^{p} \cdot y, \epsilon^{2 p} \cdot y, \epsilon^{3 p} \cdot y, \epsilon^{4 p} \cdot y$ and their orientations are given by

$$
\begin{array}{lll}
y & \rightarrow\left(L^{+},[\mathfrak{f}, v] ;\right. & \left.\epsilon^{p} L^{+},\left[-\mathfrak{f}, \epsilon^{p} v\right]\right) \\
\epsilon^{p} \cdot y & \rightarrow\left(\epsilon^{p} L^{+},\left[\operatorname{det}\left(\epsilon^{p}\right) \mathfrak{f}, \epsilon^{p} \cdot v\right] ;\right. & \left.\epsilon^{2 p} L^{+},\left[-\operatorname{det}\left(\epsilon^{p}\right) \mathfrak{f}, \epsilon^{2 p} v\right]\right) \\
\epsilon^{2 p} \cdot y \rightarrow\left(\epsilon^{2 p} L^{+},\left[\operatorname{det}\left(\epsilon^{2 p}\right) \mathfrak{f}, \epsilon^{2 p} \cdot v\right] ;\right. & \left.\epsilon^{3 p} L^{+},\left[-\operatorname{det}\left(\epsilon^{2 p}\right) \mathfrak{f}, \epsilon^{3 p} v\right]\right) \\
\epsilon^{3 p} \cdot y \rightarrow\left(\epsilon^{3 p} L^{+},\left[\operatorname{det}\left(\epsilon^{3 p}\right) \mathfrak{f}, \epsilon^{3 p} \cdot v\right] ;\right. & \left.\epsilon^{4 p} L^{+},\left[-\operatorname{det}\left(\epsilon^{3 p}\right) \mathfrak{f}, \epsilon^{4 p} v\right]\right) \\
\epsilon^{4 p} \cdot y \rightarrow\left(\epsilon^{3 p} L^{+},\left[\operatorname{det}\left(\epsilon^{4 p}\right) \mathfrak{f}, \epsilon^{4 p} \cdot v\right] ;\right. & \left.L^{+},\left[-\operatorname{det}\left(\epsilon^{4 p}\right) \mathfrak{f}, v\right]\right),
\end{array}
$$

where vector $v \in L$ points inside $L^{+}$. Summing up with appropriate signs and taking care of orientations, we get the relation

$$
y+\operatorname{det}\left(\epsilon^{p}\right) \epsilon^{p} \cdot y+\operatorname{det}\left(\epsilon^{2 p}\right) \epsilon^{2 p} \cdot y+\operatorname{det}\left(\epsilon^{3 p}\right) \epsilon^{3 p} \cdot y+\operatorname{det}\left(\epsilon^{4 p}\right) \epsilon^{4 p} \cdot y=0 .
$$

The element $\epsilon^{-2 p} j$ acts on the basis $\mathfrak{e}$ of $I^{\perp}$ by the even permutation $\left(\begin{array}{l}12345 \\ 32154\end{array}\right)$ and consequently $\epsilon^{-2 p} j$ acts on the orientation of $I$ by $\operatorname{det}\left(\epsilon^{-2 p} j\right)$. As we have seen, $\epsilon^{-2 p} j$ acts on the orientation of $L$ by $-\operatorname{det}\left(\epsilon^{-2 p} j\right)$. Also observe that $\epsilon^{-2 p} j L^{+}=$ $L^{-}$. Thus the geometric representative of the class $\epsilon^{-2 p} j \cdot y$ has orientation given by

$$
\begin{array}{ll}
\left(\epsilon^{-2 p} j L^{+},\left[\operatorname{det}\left(\epsilon^{-2 p} j\right) \mathfrak{f}, \epsilon^{-2 p} j \cdot v\right] ;\right. & \left.\epsilon^{-2 p} j \epsilon^{p} L^{+},\left[-\operatorname{det}\left(\epsilon^{-2 p} j\right) \mathfrak{f}, \epsilon^{-2 p} j \epsilon^{p} v\right]\right)= \\
\left(L^{-},\left[\operatorname{det}\left(\epsilon^{-2 p} j\right) \mathfrak{f}, \epsilon^{-2 p} j \cdot v\right] ;\right. & \left.\epsilon^{p} L^{-},\left[-\operatorname{det}\left(\epsilon^{-2 p} j\right) \mathfrak{f}, \epsilon^{-2 p} j \epsilon^{p} v\right]\right) .
\end{array}
$$

This implies the relation

$$
y-\operatorname{det}\left(\epsilon^{-2 p} j\right) \epsilon^{-2 p} j \cdot y=x-\operatorname{det}\left(\epsilon^{p}\right) \epsilon^{p} \cdot x .
$$

The reader may find Figure 9 useful as a guide for these calculations. Thus we have

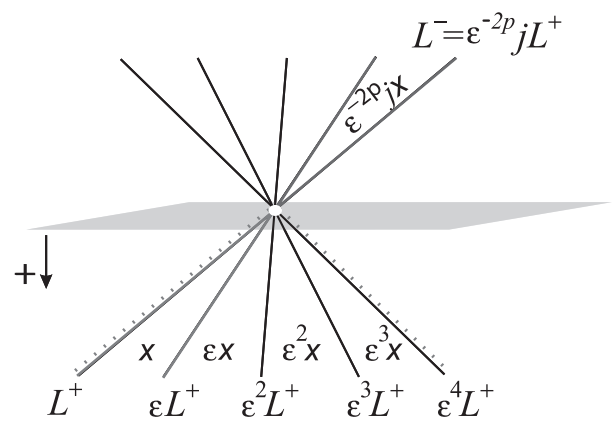

FiguRE 9. Moving of the element $y$ along the action

an isomorphism of $Q_{4 n}$-modules

$$
H_{n-4}(\bigcup \widehat{\mathcal{A}}(\alpha), \mathbb{Z}) \cong \mathbb{Z}\left[Q_{4 n}\right] x \oplus \mathbb{Z}\left[Q_{4 n}\right] y / A,
$$

where

$$
A=\left\langle\begin{array}{l}
\left(\operatorname{det}\left(\epsilon^{-2 p} j\right) \epsilon^{-2 p} j+1\right) x,\left(\epsilon^{n}-1\right) x,\left(j^{2}-1\right) y \\
\left(1+\operatorname{det}\left(\epsilon^{p}\right) \epsilon^{p}+\operatorname{det}\left(\epsilon^{2 p}\right) \epsilon^{2 p}+\operatorname{det}\left(\epsilon^{3 p}\right) \epsilon^{3 p}+\operatorname{det}\left(\epsilon^{4 p}\right) \epsilon^{4 p}\right) y \\
\left(1-\operatorname{det}\left(\epsilon^{-2 p} j\right) \epsilon^{-2 p} j\right) y+\left(\operatorname{det}\left(\epsilon^{p}\right) \epsilon^{p}-1\right) x
\end{array}\right\rangle
$$


Unfortunately, to describe the $Q_{4 n}$-module structure on $\operatorname{Hom}\left(H_{n-4}(\bigcup \widehat{\mathcal{A}}(\alpha), \mathbb{Z}), \mathbb{Z}\right)$ we have to work with the concrete basis of $H_{n-4}(\bigcup \widehat{\mathcal{A}}(\alpha), \mathbb{Z})$. Let $\Lambda=\{x, \epsilon$. $\left.x, \ldots, \epsilon^{n-1} \cdot x, y, \epsilon \cdot y, \ldots, \epsilon^{4 p-1} \cdot y\right\}$ be a basis of the free abelian group $H_{n-4}(\bigcup \widehat{\mathcal{A}}(\alpha), \mathbb{Z})$. Let $\xi \in \operatorname{Hom}\left(H_{n-4}(\bigcup \widehat{\mathcal{A}}(\alpha), \mathbb{Z}), \mathbb{Z}\right)$ be an element given on the basis $\Lambda$ by

$$
\xi(x)=1, \xi(y)=1,(\forall z \in \Lambda \backslash\{x, y\}) \xi(z)=0 .
$$

Let $\Pi=1+\epsilon^{p}+\epsilon^{2 p}+\epsilon^{3 p}+\epsilon^{4 p}+j+\epsilon^{p} j+\epsilon^{2 p} j+\epsilon^{3 p} j+\epsilon^{4 p} j$. Using the definition of the "*"-action (4.10), we compute $\Pi * \xi$ on elements of the basis $\Lambda$. It can be checked that $\Pi * \xi=0$. For example, for the basis element $x$ we have

$$
\begin{aligned}
(\Pi * \xi)(x) & =\sum_{i=0}^{4} \epsilon^{i p} * \xi(x)+\sum_{i=0}^{4} \epsilon^{i p} j * \xi(x) \\
& =\xi\left(\sum_{i=0}^{4} \operatorname{det}\left(\epsilon^{i p}\right) \epsilon^{i p} \cdot x+\sum_{i=0}^{4} \operatorname{det}\left(\epsilon^{i p} j\right) \epsilon^{i p} j \cdot x\right) \\
& =\sum_{i=0}^{4} \operatorname{det}\left(\epsilon^{i p}\right) \xi\left(\epsilon^{i p} \cdot x\right)+\sum_{i=0}^{4} \operatorname{det}\left(\epsilon^{i p} j\right) \xi\left(\epsilon^{i p} j \cdot x\right) \\
& =\xi(x)+\operatorname{det}\left(\epsilon^{3 p} j\right) \xi\left(\epsilon^{3 p} j \cdot x\right) .
\end{aligned}
$$

Since $\epsilon^{3 p} j \cdot x$ is not a basis element, $\xi$ is evaluated via the relation (4.13):

$(\Pi * \xi)(x)=\xi(x)+\operatorname{det}\left(\epsilon^{3 p} j\right) \xi\left(\epsilon^{3 p} j \cdot x\right)=\xi(x)+\operatorname{det}\left(\epsilon^{3 p} j\right) \xi\left(-\operatorname{det}\left(\epsilon^{3 p} j\right) x\right)=0$.

For the basis element $y$, applying relation (4.14) we obtain

$$
(\Pi * \xi)(y)=\xi\left(\sum_{i=0}^{4} \operatorname{det}\left(\epsilon^{i p}\right) \epsilon^{i p} \cdot y+\sum_{i=0}^{4} \operatorname{det}\left(\epsilon^{i p} j\right) \epsilon^{i p} j \cdot y\right)=0 .
$$

Now it is not hard to deduce that there is a $Q_{4 n}$-module isomorphism

$$
\operatorname{Hom}\left(H_{n-4}(\bigcup \widehat{\mathcal{A}}(\alpha), \mathbb{Z}), \mathbb{Z}\right) \cong \mathbb{Z}\left[Q_{4 n}\right] \xi / K,
$$

where

$\left.K=\left\langle 1+\epsilon^{p}+\epsilon^{2 p}+\epsilon^{3 p}+\epsilon^{4 p}+j+\epsilon^{p} j+\epsilon^{2 p} j+\epsilon^{3 p} j+\epsilon^{4 p} j\right) \xi,\left(j^{2}-1\right) \xi,\left(\epsilon^{n}-1\right) \xi\right\rangle$.

(D) Let $x \in H_{n-4}(\bigcup \widehat{\mathcal{A}}(\alpha), \mathbb{Z})$ be the homology class induced by $L$. We analyze the set equality $\epsilon^{-3} j \cdot L=L$. Let $\mathfrak{e}=\left[e_{1}, e_{2}, e_{3}, e_{4}+e_{5}+e_{6}\right]$ be the fixed basis of $L^{\perp}$. Then $\epsilon^{-3} j$ acts on $\mathfrak{e}$ as the odd permutation $\left(\begin{array}{l}1234 \\ 3214\end{array}\right)$. This implies the equality $x=-\operatorname{det}\left(\epsilon^{-3} j\right) \epsilon^{-3} j \cdot x$. Also, $j^{2} \cdot x=\epsilon^{6} \cdot x=x$.

Let us denote by $L^{+}$and $(\epsilon L)^{+}$the following half-spaces:

$$
L^{+}=\left\{x \in L \mid x_{4} \geq 0\right\} \text { and }(\epsilon L)^{+}=\left\{x \in \epsilon L \mid x_{1} \geq 0\right\} .
$$

In contrast to the previous situation $(\mathrm{C})$, here we have $\epsilon \cdot L^{+} \neq(\epsilon L)^{+}$. Let $y \in H_{n-4}(\bigcup \widehat{\mathcal{A}}(\alpha), \mathbb{Z})$ be the homology class induced by $L^{+} \cup(\epsilon L)^{+}$. Again the set equality $\epsilon^{-2} j \cdot\left(L^{+} \cup(\epsilon L)^{+}\right)=L^{+} \cup(\epsilon L)^{+}$is the starting point of our calculation. The element $\epsilon^{-2} j$ acts on the basis $\left[e_{1}, e_{2}, e_{3}, e_{4}, e_{5}+e_{6}\right]$ of $(L \cap \epsilon L)^{\perp}$ as the even permutation $\left(\begin{array}{l}12345 \\ 43215\end{array}\right)$. Thus $\epsilon^{-2} j$ acts on the orientation of $L \cap \epsilon L$ as the multiplication with $\operatorname{det}\left(\epsilon^{-2} j\right)$. Let $\mathfrak{f}$ be the basis of $L \cap \epsilon L$. We choose the following orientation of the geometric representative of the element $y$ :

$$
\left(L^{+},[\mathfrak{f}, v] ;(\epsilon L)^{+},\left[-\mathfrak{f}, \epsilon^{-2} j \cdot v\right]\right),
$$


where $v \in L$ points inside $L^{+}$. The vector $\epsilon^{-2} j \cdot v$ points inside $(\epsilon L)^{+}$. The action of $\epsilon^{-2} j$ on fixed orientations provides

$$
\left((\epsilon L)^{+},\left[\operatorname{det}\left(\epsilon^{-2} j\right) \mathfrak{f}, \epsilon^{-2} j \cdot v\right] ; L^{+},\left[-\operatorname{det}\left(\epsilon^{-2} j\right) \mathfrak{f}, v\right]\right)
$$

because $\epsilon^{-2} j L^{+}=(\epsilon L)^{+}$and $\epsilon^{-2} j(\epsilon L)^{+}=L^{+}$. Therefore,

$$
y+\operatorname{det}\left(\epsilon^{-2} j\right) \epsilon^{-2} j \cdot y=0 .
$$

Again, $j^{2} \cdot y=\epsilon^{6} \cdot y=y$.

Let us denote by $F_{1}, \ldots, F_{6}$ the following convex cones:

$$
\begin{array}{ll}
F_{1}=\left\{x \in L \mid x_{4} \geq 0, x_{6} \geq 0\right\}, & F_{2}=\left\{x \in \epsilon L \mid x_{5} \geq 0, x_{1} \geq 0\right\}, \\
F_{3}=\left\{x \in \epsilon^{2} L \mid x_{6} \geq 0, x_{2} \geq 0\right\}, & F_{4}=\left\{x \in \epsilon^{3} L \mid x_{1} \geq 0, x_{3} \geq 0\right\}, \\
F_{5}=\left\{x \in \epsilon^{4} L \mid x_{2} \geq 0, x_{4} \geq 0\right\}, & F_{6}=\left\{x \in \epsilon^{5} L \mid x_{3} \geq 0, x_{5} \geq 0\right\} .
\end{array}
$$

Let $z \in H_{n-4}(\cup \widehat{\mathcal{A}}(\alpha), \mathbb{Z})$ be the element geometrically represented by the union $S=F_{1} \cup \ldots \cup F_{6}$. Then $g \cdot S=S$ for every $g \in Q_{4 n}$. Let us fix a basis for each of the convex cones by

$$
\begin{aligned}
& F_{1} \rightarrow\left[e_{4}, e_{6}\right], \quad F_{2} \rightarrow\left[e_{5}, e_{1}\right], \quad F_{3} \rightarrow\left[e_{6}, e_{2}\right], \\
& F_{4} \rightarrow\left[e_{1}, e_{3}\right], \quad F_{5} \rightarrow\left[e_{2}, e_{4}\right], \quad F_{6} \rightarrow\left[e_{3}, e_{5}\right] .
\end{aligned}
$$

Since the element $\epsilon$ does not change the orientation of $S$, we have $\epsilon \cdot z-z=0$. On the other hand, the element $j$ does change the orientation of $S$ and so $j \cdot z+z=0$. Figure 10 may be used as a guide for visualizing geometric representatives of classes $x, y$ and $z$. Let $\Lambda=\left\{x, \epsilon \cdot x, \ldots, \epsilon^{5} \cdot x, y, \epsilon \cdot y, \ldots, \epsilon^{5} \cdot y, z\right\}$ be a basis of the free abelian
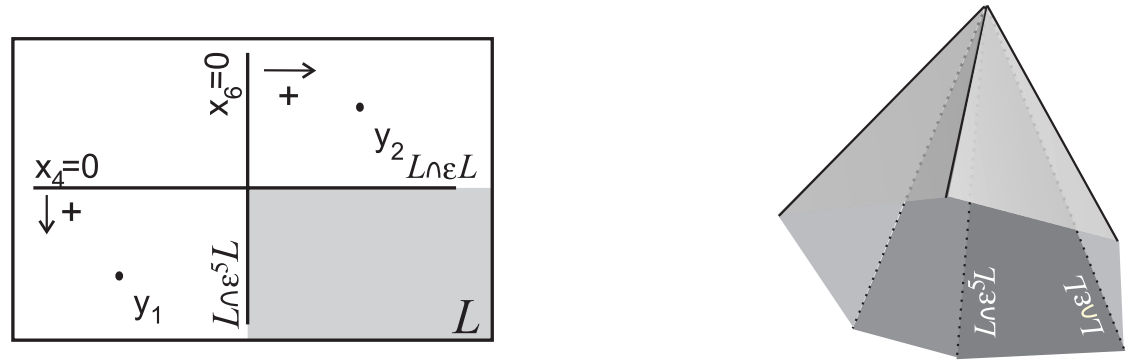

FiguRE 10. The geometric representatives in the case $(1,1,1,3)$

group $H_{n-4}(\bigcup \widehat{\mathcal{A}}(\alpha), \mathbb{Z})$. Let $\xi, \eta, \zeta \in \operatorname{Hom}\left(H_{n-4}(\bigcup \widehat{\mathcal{A}}(\alpha), \mathbb{Z}), \mathbb{Z}\right)$ be the elements given on the basis $\Lambda$ by

$$
\begin{aligned}
& \xi(x)=1,(\forall t \in \Lambda \backslash\{x\}) \xi(t)=0 \\
& \eta(y)=1,(\forall t \in \Lambda \backslash\{y\}) \eta(t)=0 \\
& \zeta(y)=1,(\forall t \in \Lambda \backslash\{z\}) \zeta(t)=0 .
\end{aligned}
$$

By direct computation on the basis $\Lambda$ one can prove that

$$
\epsilon^{-3} j \cdot \xi+\xi=0, \eta+\epsilon^{-2} j \cdot \eta=0, \epsilon \cdot \zeta+\zeta=0, \epsilon \cdot \zeta-\zeta=0,
$$

because $\operatorname{det}(\epsilon)=-1$ and $\operatorname{det}(j)=1$.

(E) The proof follows in the footsteps of the previous cases. Let $x \in$ $H_{n-4}(\bigcup \widehat{\mathcal{A}}(\alpha), \mathbb{Z})$ be the homology class induced by $L$. The set equality $L=\epsilon^{-p} j L$ 
implies the relation $\left(1+\operatorname{det}\left(\epsilon^{-p} j\right) \epsilon^{-p} j\right) \cdot x=0$. Let $y \in H_{n-4}(\bigcup \widehat{\mathcal{A}}(\alpha), \mathbb{Z})$ be the homology class induced by the union of the following half-spaces:

$$
L^{+}=\left\{x \in L \mid x_{3 q+1}+\ldots+x_{4 p} \geq 0\right\},\left(\epsilon^{q} L\right)^{+}=\left\{x \in \epsilon^{q} L \mid x_{1}+\ldots+x_{p} \geq 0\right\} .
$$

Again $\epsilon^{q} L^{+} \neq\left(\epsilon^{q} \cdot L\right)^{+}$. The set equality $\epsilon^{-2 p-2 q} j\left(L^{+} \cup\left(\epsilon^{q} L\right)^{+}\right)=L^{+} \cup\left(\epsilon^{q} L\right)^{+}$, with careful orientation calculation, implies the relation $\left(1+\operatorname{det}\left(\epsilon^{-2 p-2 q} j\right) \epsilon^{-2 p-2 q} j\right)$. $y=0$.

If we use the basis $\Lambda=\left\{x, \epsilon \cdot x, \ldots, \epsilon^{n-1} \cdot x, y, \epsilon \cdot y, \ldots, \epsilon^{n-1} \cdot y\right\}$ for $H_{n-4}(\bigcup \widehat{\mathcal{A}}(\alpha), \mathbb{Z})$, and define $\xi, \eta \in \operatorname{Hom}\left(H_{n-4}(\bigcup \widehat{\mathcal{A}}(\alpha), \mathbb{Z}), \mathbb{Z}\right)$ by

$$
\begin{aligned}
& \xi(x)=1,(\forall t \in \Lambda \backslash\{x\}) \xi(t)=0 \\
& \eta(y)=1,(\forall t \in \Lambda \backslash\{y\}) \eta(t)=0
\end{aligned}
$$

the result follows.

(F) The statement follows as in the case (E) using the set equalities $L=$ $\epsilon^{-(p+q)} j L$ and $L \cap \epsilon^{p+q} L=j\left(L \cap \epsilon^{p+q} L\right)$.

(I) Let $x \in H_{n-4}(\bigcup \widehat{\mathcal{A}}(\alpha), \mathbb{Z})$ be the homology class induced by $L$. The element $\epsilon^{-2 q} j$ acts on the basis of $L^{\perp}$ by an even permutation. Thus the set equality $\epsilon^{-2 q} j L=L$ transforms into the relation $\left(\left(\operatorname{det} \epsilon^{-2 q} j\right) \epsilon^{-2 q} j-1\right) \cdot x=$ 0 . For a basis $\Lambda=\left\{x, \epsilon \cdot x, \ldots, \epsilon^{n-1} \cdot x\right\}$ of $H_{n-4}(\bigcup \widehat{\mathcal{A}}(\alpha), \mathbb{Z})$, we define $\xi \in$ $\operatorname{Hom}\left(H_{n-4}(\bigcup \widehat{\mathcal{A}}(\alpha), \mathbb{Z}), \mathbb{Z}\right)$ on $\Lambda$ by

$$
\xi(x)=1,(\forall t \in \Lambda \backslash\{x\}) \xi(t)=0 .
$$

Then

$$
\begin{aligned}
\epsilon^{-2 q} j * \xi\left(\epsilon^{i} \cdot x\right) & =\operatorname{det}\left(\epsilon^{-2 q} j\right) \xi\left(j \epsilon^{2 q} \epsilon^{i} \cdot x\right)=\operatorname{det}\left(\epsilon^{-2 q} j\right) \xi\left(\epsilon^{-2 q-i} j \cdot x\right) \\
& =\operatorname{det}\left(\epsilon^{-2 q} j\right) \xi\left(\epsilon^{-i} \cdot\left(\epsilon^{-2 q} j \cdot x\right)\right)=\xi\left(\epsilon^{-i} \cdot x\right) \\
& =\xi\left(\epsilon^{i} \cdot x\right) .
\end{aligned}
$$

The last equality follows from the definition of $\xi$. Thus, $\epsilon^{-2 q} j * \xi=\xi$.

(J) Let $x \in H_{n-4}(\bigcup \widehat{\mathcal{A}}(\alpha), \mathbb{Z})$ be the homology class induced by $L$. The element $\epsilon^{p} j$ acts on $L^{\perp}$ by an odd permutation $\left(\begin{array}{l}1234 \\ 3214\end{array}\right)$. Thus the set equality $\epsilon^{-2 q} j L=$ $L$ transforms to the relation $\left(\operatorname{det}\left(\epsilon^{-2 q} j\right) \epsilon^{-2 q} j+1\right) \cdot x=0$ in $H_{n-4}(\bigcup \widehat{\mathcal{A}}(\alpha), \mathbb{Z})$. Like in the previous cases for the particular choice of an element $\xi \in$ $\operatorname{Hom}\left(H_{n-4}(\bigcup \widehat{\mathcal{A}}(\alpha), \mathbb{Z}), \mathbb{Z}\right)$ we obtain the relation $\left(\epsilon^{-2 q} j+1\right) * \xi=0$.

(M) In all the remaining cases there are no nontrivial relations except

$$
\epsilon^{n}=j^{2}=1 \text {. }
$$

The knowledge of the $Q_{4 n}$-module structure on $H_{2}(M ; \mathbb{Z})$ allows us to read off the group of coinvariants.

Corollary 4.9. With the assumptions of Theorem 4.8 the corresponding groups of coinvariants are the following:

(A) If $\alpha=(p, p, p, p)$, then $H_{2}(M ; \mathbb{Z})_{Q_{4 n}} \cong \mathbb{Z}_{2}$.

(B) If $\alpha=(p, q, p, q)$ and $p \neq q$, then $H_{2}(M ; \mathbb{Z})_{Q_{4 n}} \cong \mathbb{Z}_{2}$.

(C) If $\alpha=(p, p, p, 2 p)$, then $H_{2}(M ; \mathbb{Z})_{Q_{4 n}} \cong \mathbb{Z}_{10}$.

(D) If $\alpha=(1,1,1,3)$, then $H_{2}(M ; \mathbb{Z})_{Q_{4 n}} \cong \mathbb{Z}_{2} \oplus \mathbb{Z}_{2} \oplus \mathbb{Z}_{2}$.

(E) If $\alpha=(q, q, q, p), p \notin\{q, 2 q\}$ and $3 q+p>6$, then $H_{2}(M ; \mathbb{Z})_{Q_{4 n}} \cong \mathbb{Z}_{2} \oplus \mathbb{Z}_{2}$.

(F) If $\alpha=(p, q, p, p+q)$ and $p \neq q$, then $H_{2}(M ; \mathbb{Z})_{Q_{4 n}} \cong \mathbb{Z}_{2} \oplus \mathbb{Z}_{2}$. 
(G) If $\alpha=(p, p, 2 p, 2 p)$, then $H_{2}(M ; \mathbb{Z})_{Q_{4 n}} \cong$ ?.

(H) If $\alpha=(p, q, p+q, p+q)$ and $p \neq q$, then $H_{2}(M ; \mathbb{Z})_{Q_{4 n}} \cong$ ?.

(I) If $\alpha=(p, p, q, q)$ and $p \neq q, p \neq 2 q, q \neq 2 p$, then $H_{2}(M . \mathbb{Z})_{Q_{4 n}} \cong \mathbb{Z}$.

(J) If $\alpha=(p, q, r, q)$ and $r \notin\{q, p, p+q\}$, then $H_{2}(M ; \mathbb{Z})_{Q_{4 n}} \cong \mathbb{Z}_{2}$.

(K) If $\alpha=(p, q, r, p+q)$ and $r \notin\{p, p+q\}$, then $H_{2}(M ; \mathbb{Z})_{Q_{4 n}} \cong$ ?.

(L) If $\alpha=(p, p, q, q+r)$, and $p \neq q, r \neq 0$, then $H_{2}(M ; \mathbb{Z})_{Q_{4 n}} \cong$ ?.

(M) In all the remaining cases $H_{2}(M ; \mathbb{Z})_{Q_{4 n}} \cong \mathbb{Z}$.

4.4. The main theorem. This section is central to the paper. We gather together all the information collected in previous sections and use it to obtain a reasonably complete answer to Problem 2.3.

Theorem 4.10. Suppose that $Q_{4 n}$ is a generalized quaternion group and $M(\alpha)$ the complement of the arrangement $\mathcal{A}(\alpha)$ introduced in Section 2.2. Then our knowledge about the existence of $Q_{4 n}$-equivariant maps $F: S^{3} \rightarrow M(\alpha)$ (Problem 2.3) can be summarized as follows.

(A) If $\alpha=(p, p, p, p)$ or $\alpha=(p, p, p, 2 p)$, then there are no $Q_{4 n}$-equivariant maps $F: S^{3} \rightarrow M(\alpha)$.

(B) If $\alpha$ is not of the form $(p, q, p+q, p+q)$, or ( $p, q, r, p+q), r \notin\{p, p+q\}$, or $(p, p, q, q+r)$, then there exists a $Q_{4 n}$-map $F: S^{3} \rightarrow M(\alpha)$.

(C) If $\alpha=(p, q, p+q, p+q)$, or $\alpha=(p, q, r, p+q), r \notin\{p, p+q\}$, or $\alpha=(p, p, q, q+r)$, the methods of the paper are inconclusive and a further analysis is needed.

Proof. (A.1) Let $\alpha=(p, p, p, p)$. In order to prove that $\left[d_{Q_{4 n}}(h)(e)\right]=\left[\left\|y_{1}\right\|\right] \neq 0$ we rely on the isomorphism (4.8),

$$
\phi: H_{2}(M(\mathcal{A}(\alpha)), \mathbb{Z}) \rightarrow \operatorname{Hom}\left(H_{n-4}(\bigcup \widehat{\mathcal{A}}(\alpha), \mathbb{Z}), \mathbb{Z}\right) .
$$

Using the fact that this isomorphism is related to the linking number and that $y_{1} \in L \backslash \bigcup_{g \in Q_{4 n}: g L \neq L} g L$, we have that $\left(\widehat{y_{1}+L^{\perp}}\right)$ links only with $\widehat{L}$, and therefore on the basis $\Lambda$,

$$
\phi\left(\left\|y_{1}\right\|\right)\left(\epsilon^{i} \cdot x\right)=\operatorname{link}\left(\widehat{\epsilon^{i} L},\left(\widehat{y_{1}+L^{\perp}}\right)\right)= \begin{cases}1, & i=0, \\ 0, & \text { otherwise. }\end{cases}
$$

Here $\widehat{\epsilon^{i} L}$ and $\left(\widehat{y_{1}+L^{\perp}}\right)$ are respectively one-point compactifications of the linear subspace $\epsilon^{i} L$ and the affine subspace $y_{1}+L^{\perp}$ in the sphere $\widehat{W_{n}} \approx S^{n-1}$. Thus $\phi\left(\left\|y_{1}\right\|\right)=\xi$.

The obstruction element $d_{Q_{4 n}}(h)(e)=\left\|y_{1}\right\|$ is mapped to the generator of the group $H_{2}(M ; \mathbb{Z})_{Q_{4 n}} \cong \mathbb{Z}_{2}$ by the quotient map $H_{2}(M ; \mathbb{Z}) \rightarrow H_{2}(M ; \mathbb{Z})_{Q_{4 n}}$. Therefore, there is no $Q_{4 n}$-map $F: S^{3} \rightarrow M(\alpha)$.

(A.2) Let $\alpha=(p, p, p, 2 p)$. Then $d_{Q_{4 n}}(h)(e)=4\left\|y_{1}\right\|$, by Theorem 4.3, (B). Like in (A.1) we compute the $\phi$ image of the point class $\left\|y_{1}\right\|$. Since $y_{1} \in L^{+}$(see (4.3) and Theorem $4.8(\mathrm{C})$ ) we have on the basis $\Lambda$,

$$
\begin{aligned}
& \phi\left(\left\|y_{1}\right\|\right)\left(\epsilon^{i} \cdot x\right)=\operatorname{link}\left(\widehat{\epsilon^{i} L},\left(\widehat{y_{1}+L^{\perp}}\right)\right)= \begin{cases}1, & i=0, \\
0, & \text { otherwise, }\end{cases} \\
& \phi\left(\left\|y_{1}\right\|\right)\left(\epsilon^{j} \cdot y\right)=\operatorname{link}\left(\epsilon^{j}\left(\widehat{L^{+\cup \epsilon^{p}}} L^{+}\right),\left(\widehat{y_{1}+L^{\perp}}\right)\right)= \begin{cases}1, & j=0, \\
0, & \text { otherwise. }\end{cases}
\end{aligned}
$$


Consequently, $\phi\left(\left\|y_{1}\right\|\right)=\xi \in \operatorname{Hom}\left(H_{n-4}(\bigcup \widehat{\mathcal{A}}(\alpha), \mathbb{Z}), \mathbb{Z}\right)$. Thus, $\left[d_{Q_{4 n}}(h)(e)\right]=$ $4 \cdot\left\|y_{1}\right\| \in H_{2}(M ; \mathbb{Z})_{Q_{4 n}} \cong \mathbb{Z}_{10}$ is a nonzero element $4 *$ (generator). Therefore, there are no $Q_{4 n}$-maps $F: S^{3} \rightarrow M(\alpha)$.

(B) Assume that $\alpha$ is not of the form $(p, p, p, p),(p, p, p, 2 p),(p, q, p+q, p+q)$, $(p, q, r, p+q), r \notin\{p, p+q\}$ or $(p, p, q, q+r)$. Then the cohomology class of the obstruction cocycle $\left[d_{Q_{4 n}}(h)(e)\right]$ is divisible by 2 (Theorem 4.3). On the other hand this cohomology class is a torsion element of $H_{2}(M ; \mathbb{Z})_{Q_{4 n}}$ (Lemma 4.1). Since Corollary 4.9 implies that the only torsion summands which can appear are copies of $\mathbb{Z}_{2}$, we conclude that $\left[d_{Q_{4 n}}(h)(e)\right]=0$. Thus a $Q_{4 n}$-map $F: S^{3} \rightarrow M(\alpha)$ must exist in all these cases.

4.5. Concluding remarks. Perhaps one of the main general problems of the emerging field of Computational Topology is to determine the scope of the field and major lines of prospective research. This includes the identification of classes of model problems where topology and computational mathematics interact in an essential way.

One of our objectives in this paper was to demonstrate that the calculation of the equivariant obstruction for the existence of an equivariant map is one such problem. The algorithms used in these calculations are based on important results of topology and geometric combinatorics, including the Goresky-MacPherson formula for the homology of the complement of an affine arrangement, equivariant obstruction exact sequence, etc. However, the crux of the computation is in the introduction and manipulation of the so-called "point" and "broken point" homology classes, which are not only of theoretical interest but allow quite efficient calculations, one step away from an effective computer implementation.

The paper focuses on the currently active problem involving $G$-equivariant maps where $G$ is a subgroup of the dihedral group $D_{2 n}$, alternatively the generalized quaternion group $Q_{4 n}$, but the technique is quite general and may be adapted to other problems of a similar nature.

\section{ACKNOWLEDGMENTS}

We are grateful to I. Bárány, S. Fomin, C. Schultz and G. Zigler. The referee made the enormous effort to improve all aspects of the paper, and we are very grateful for all the suggestions and observations. We learned a great deal from the referee.

\section{REFERENCES}

[1] J. Akiyama, A. Kaneko, M. Kano, G. Nakamura, E. Rivera-Campo, S. Tokunaga, and J. Urrutia, Radial perfect partitions of convex sets in the plane. In Discrete and Computational Geometry (J. Akiyama et al., eds.), Lect. Notes Comput. Sci. 1763, pp. 1-13. Springer, Berlin, 2000. MR 1787512

[2] I. Bárány, Geometric and combinatorial applications of Borsuk's theorem, János Pach, ed., Algorithms and Combinatorics 10, Springer-Verlag, Berlin, 1993.

[3] I. Bárány and J. Matoušek, Simultaneous partitions of measures by $k$-fans, Discrete Comput. Geom., 25: 317-334, 2001. MR.1815435 (2002c:52016)

[4] I. Bárány and J. Matoušek, Equipartitions of two measures by a 4-fan, Discrete Comput. Geom., 27 : 293-302, 2002. MR1921557 (2003f:28008)

[5] S. Bespamyatnikh, D. Kirkpatrick, and J. Snoeyink, Generalizing ham sandwich cuts to equitable subdivisions. Discrete Comput. Geom., 24:605-622, 2000. MR 1799604(2001j:52012)

[6] A. Björner, Topological methods, In R. Graham, M. Grötschel, and L. Lovász, editors, Handbook of Combinatorics. North-Holland, Amsterdam, 1995. MR:1373690 (96m:52012) 
[7] P. Blagojević, The partition of measures by 3-fans and computational obstruction theory, arXiv: math.CO/ 0402400, 2004

[8] P. Blagojević and A. Dimitrijevic Blagojević, Using Equivariant Obstruction Theory in Combinatorial Geometry, Topology and its Applications, 154, 2635-2655, 2007, http://dx.doi.org/10.1016/j.topol.2007.04.007. MR2340948

[9] G.E. Bredon, Topology and Geometry, Graduate Texts in Mathematics 139, Springer, 1995. MR1700700 (2000b:55001)

[10] K.S. Brown, Cohomology of groups, Springer-Verlag, New York, Berlin, 1982. MR672956 (83k:20002)

[11] H. Cartan and S. Eilenberg, Homological Algebra, Princeton University Press, 1956. MR0077480 (17:1040e)

[12] P.E. Conner and E.E. Floyd, Differentiable periodic maps, Springer-Verlag, Berlin, 1964. MR0176478 (31:750)

[13] T. tom Dieck, Transformation groups, de Gruyter Studies in Math. 8, Berlin, 1987. MR:889050 (89c:57048)

[14] A. Dold, Lectures on Algebraic Topology, Springer-Verlag, 1980. MR606196 (82c:55001)

[15] M. Goresky and R. MacPherson, Stratified Morse Theory, Springer, 1988. MR.932724 (90d:57039)

[16] M. Gromov, Partial differential relations, Springer-Verlag (1986), Ergebnisse der Mathematik und ihrer Grenzgebiete, 3 Folge, Vol. 9. MR864505 (90a:58201)

[17] H. Ito, H. Uehara, and M. Yokoyama, 2-dimension ham-sandwich theorem for partitioning into three convex pieces, In Discrete and Computational Geometry (J. Akiyama et. al eds.), Lect. Notes Comput. Sci. 1763, pp. 129-157. Springer, Berlin 2000. MR.1787521

[18] A. Kaneko and M. Kano, Balanced partitions of two sets of points in the plane, Comput. Geom. Theor. Appl., 13(4), 253-261, 1999. MR1719059 (2001i:52001)

[19] J. Matoušek, Using the Borsuk-Ulam Theorem; Lectures on Topological Methods in Combinatorics and Geometry. Springer, Berlin, 2003. MR 1988723(2004i:55001)

[20] J. R. Munkres, Elements of Algebraic Topology, Addison-Wesley, $1984 . \quad$ MR755006 (85m:55001)

[21] P. Orlik and H. Terao, Arrangements of Hyperplanes, Grundlehren der mathematischen Wissenschaften 300, Springer-Verlag, 1992. MR1217488 (94e:52014)

[22] J. Pach (Ed.), New Trends in Discrete and Computational Geometry, Algorithms and Combinatorics 10, Springer 1993. MR1228036 (94b:52001)

[23] E. Ramos, Equipartitions of mass distributions, by hyperplanes, Discrete Comput. Geom., 15 : 147-167, 1996. MR.1368272 (97e:52021)

[24] Referee report

[25] T. Sakai, Radial partitions of point sets in $R^{2}$, Manuscript, Tokoha Gakuen University, 1998.

[26] C. Schultz, Private communications.

[27] S. Sundaram and V. Welker, Group actions on arrangements of linear subspaces and applications to configuration spaces, T.A.M.S., Vol 349, No. 4, 1997, 1389-1420 MR1340186 (97h:52012)

[28] H. Tverberg and S. Vrećica, On generalizations of Radon's theorem and the ham sandwich theorem, Europ. J. Combinatorics 14, 1993, pp. 259-264. MR1215336 (94d:52004)

[29] S. Vrećica and R. Živaljević, The ham sandwich theorem revisited, Israel J. Math. 78, 1992, pp. 21-32. MR.1194956 (94e:52007)

[30] S. Vrećica and R. Zivaljević, Conical equipartitions of mass distributions, Discrete Comput. Geom., 225:335-350, 2001. MR:1815436 (2002c:52017)

[31] S. Vrećica and R. Živaljević, Arrangements, equivariant maps and partitions of measures by 4-fans, in Discrete and Computational Geometry: The Goodman-Pollack Festschrift (J. Goodman et al., eds.), pp. 829-849, Algorithms and Combinatorics 25, Springer Verlag 2003.

[32] C.T.C. Wall, Surgery on Compact Manifolds, Academic Press, 1970. MR.0431216 (55:4217)

[33] G. M. Ziegler and R. T. Živaljević, Homotopy types of subspace arrangements via diagrams of spaces, Math. Ann. 295:527-548, 1993. MR1204836 (94c:55018)

[34] R. Živaljević, Topological methods, in CRC Handbook of Discrete and Computational Geometry (new edition), J.E. Goodman, J.O'Rourke, eds. CRC Press, Boca Raton, 2004. MR2082993 (2005j:52001)

[35] R. Zivaljević, The Tverberg-Vrećica problem and the combinatorial geometry on vector bundles, Israel J. Math. 111 (1999), 53-76. MR1710731(2000h:05068) 
[36] R. Živaljević, User's guide to equivariant methods in combinatorics, Publ. Inst. Math. Belgrade, 59(73), 1996, 114-130. MR1444570 (98c:55001)

[37] R. Živaljević, User's guide to equivariant methods in combinatorics II, Publ. Inst. Math. Belgrade, 64(78), 1998, 107-132. MR1668705 (2000c:55001)

[38] R. Živaljević and S. Vrećica, An extension of the ham sandwich theorem, Bull. London Math. Soc. 22, 1990, pp. 183-186. MR 1045292 (91j:52006)

Mathematical Institute of the Serbian Academy of Sciences and Arts, Belgrade, SERBIA

E-mail address: pavleb@mi.sanu.ac.yu

Mathematical Faculty, University of Belgrade, Belgrade, Serbia

E-mail address: vrecica@matf.bg.ac.yu

Mathematical Institute of the Serbian Academy of Sciences and Arts, Belgrade, SERBIA

E-mail address: rade@mi.sanu.ac.yu 